\title{
Dynamic Analysis of the Nonlinear Chaotic System with Multistochastic Disturbances
}

\author{
Lingling Geng, Yongguang Yu, and Shuo Zhang \\ Department of Mathematics, Beijing Jiaotong University, Beijing 100044, China \\ Correspondence should be addressed to Yongguang Yu; ygyu@bjtu.edu.cn
}

Received 12 April 2014; Revised 19 June 2014; Accepted 30 June 2014; Published 6 August 2014

Academic Editor: Miguel A. F. Sanjuan

Copyright (C) 2014 Lingling Geng et al. This is an open access article distributed under the Creative Commons Attribution License, which permits unrestricted use, distribution, and reproduction in any medium, provided the original work is properly cited.

\begin{abstract}
The nonlinear chaotic system with multistochastic disturbances is investigated. Based on the orthogonal polynomial approximation, the method of transforming the system into an equivalent deterministic system is given. Then dynamic analysis of the nonlinear chaotic system with multistochastic disturbances can be reduced into that of its equivalent deterministic system. Especially, the Lorenz system with multistochastic disturbances is studied to demonstrate the feasibility of the given method. And its dynamic behaviors are gained including the phase portrait, the bifurcation diagram, the Poincare section, and the maximum Lyapunov exponent.
\end{abstract}

\section{Introduction}

Chaos is a hot topic in the nonlinear science. And it has been found to be useful and has great potential in many disciplines such as mixing liquids with low power consumption, presenting outages in power systems, biomedical engineering applications involving signals from the brain and heart, to name just a few [1]. The deterministic chaotic system has been studied extensively [2-5]. However, stochastic processes are prevalent in nature. They affect all physical phenomena from both external and internal sources. For example, noise in physics, chemistry, and biology has a profound effect on the chaotic dynamical system; strong wind can cause nonlinear random vibration of large bridges and offshore platforms. Hence, a growing number of scholars have shown great interests in the research of nonlinear stochastic dynamics [69].

To research the dynamic behaviors of the nonlinear stochastic systems, there are several mathematical methods available: Monte Carlo method [10], stochastic finite element method [11, 12], and orthogonal polynomial approximation method $[13,14]$. Monte Carlo method is simple and universal but usually involved a quite amount of computation. Stochastic finite element method involved the least computation but is usually restricted to system with random variables of small perturbation. Based on the expansion theory of orthogonal polynomials [15-19], the orthogonal polynomial approximation without the limitation of small perturbation was introduced in [13] and improved in [14]. Recently, the orthogonal polynomial approximation has been successfully applied for studying the dynamic behaviors of the nonlinear systems with one random parameter [20-24]. In 2003, the evolutionary random response problems of the linear systems with random parameters were firstly studied $[20,21]$ by Chebyshev polynomial and Gegenbauer polynomial. The period-doubling bifurcation in double-well stochastic Duffing system was analyzed in [22]. The bifurcation in an extended Van der Pol system with bounded random parameter was discussed in [23]. And the control problems of stochastic Duffing-Van der Pol system were researched in [24].

In the earlier literature, most of the discussions are devoted to the systems with one stochastic disturbance [2024], which have only one random parameter in their models. However, there exist all kinds of stochastic factors in the nonlinear chaotic process in the real world. In order to explore the dynamic behaviors of the nonlinear chaotic system under the influence of several kinds of stochastic factors, it is necessary and important to research the nonlinear chaotic system with multistochastic disturbances, which means that two or more parameters of the nonlinear chaotic system are designed as random ones. Moreover, the nonlinear chaotic system with multistochastic disturbances is more general than such 
system with deterministic or one random parameter. But the works on this subject are very few as far as we know [25].

Motivated by the above discussion, the nonlinear chaotic system with multistochastic disturbances is investigated in this paper. Firstly, in order to research its complex and fascinating dynamic behaviors, the method of simplifying the system into an equivalent deterministic system is given according to the orthogonal polynomial approximation. Then the dynamical characteristics of the system can be obtained by analyzing the equivalent deterministic one. For illustrating purposes, the Lorenz system with multistochastic disturbances is studied. Its dynamical characteristics are obtained by analyzing the phase portrait, the bifurcation diagram, the Poincare section, and the maximum Lyapunov exponent of the equivalent deterministic system. Finally, numerical simulation shows that dynamic behaviors in the Lorenz system with multistochastic disturbances are determined not only by system parameters, but also by multistochastic disturbances and stochastic intensities. In addition, the Lorenz system with multistochastic disturbances is more general than such system with deterministic or one random parameter.

The rest of this paper is organized as follows. The method of transforming the nonlinear chaotic system with multistochastic disturbances into an equivalent deterministic system is given in Section 2. The Lorenz system with multistochastic disturbances is discussed in Section 3. Dynamic analysis and numerical simulation are given in Section 4. And conclusion closes the paper in Section 5.

\section{Orthogonal Polynomial Approximation for the Nonlinear Chaotic System with Multistochastic Disturbances}

The nonlinear chaotic system can be described as

$$
\dot{x}(t)=f(t, x),
$$

where $x(t)=\left(x_{1}(t), x_{2}(t), \ldots, x_{n}(t)\right)^{T}$ is the state vector and $f(t, x)=\left(f_{1}(t, x), f_{2}(t, x), \ldots, f_{n}(t, x)\right)^{T}$ is a continuous nonlinear vector function. Now, consider the nonlinear chaotic system with multistochastic disturbances; that is,

$$
\dot{x}(t, \xi)=f(t, x(t, \xi))
$$

where $\xi=\left(\xi_{1}, \xi_{2}, \ldots, \xi_{m}\right)^{T}$ is a random vector and $\xi_{i}(i=$ $1,2, \ldots, m)$ are independent identically distributed random variables. They are defined on $[a, b]$ with the probability density function $\rho\left(\xi_{i}\right)$.

Remark 1 . If $m=1$ and $\xi=\xi_{1}$, then only one parameter in the system (2) is random. Then the system (2) is a nonlinear chaotic system with one stochastic disturbance. If $m \geq 2$ and $\xi=\left(\xi_{1}, \xi_{2}, \ldots, \xi_{m}\right)^{T}$, then two or more parameters in the system (2) are random. In this case, the system (2) is a nonlinear chaotic system with multistochastic disturbances. For different kinds of stochastic disturbances, we consider the perturbation of parameters in the system (2) as a random vector $\xi$ in this paper.
According to the principle of the orthogonal polynomial approximation [26], the responses of the system (2) can be approximately expressed under the condition of the convergence in mean square as follows:

$$
\begin{aligned}
x(t, \xi)=\sum_{j_{1}=0}^{N_{1}} \sum_{j_{2}=0}^{N_{2}} \cdots \sum_{j_{m}=0}^{N_{m}} x_{j_{1} j_{2} \cdots j_{m}}(t) U_{j_{1}} & \\
& \times\left(\xi_{1}\right) U_{j_{2}}\left(\xi_{2}\right) \cdots U_{j_{m}}\left(\xi_{m}\right),
\end{aligned}
$$

where $x_{j_{1} j_{2} \cdots j_{m}}(t)=\iint \cdots \int \rho\left(\xi_{1}\right) \cdots \rho\left(\xi_{m}\right) U_{j_{1}}\left(\xi_{1}\right) \cdots$ $U_{j_{m}}\left(\xi_{m}\right) x(t, \xi) d \xi_{1} \cdots d \xi_{m} . U_{j_{i}}\left(\xi_{i}\right)$ represents the $j_{i}$ th orthogonal polynomials and $N_{i}(i=1, \ldots, m)$ represents the largest order of the polynomials.

Substituting (3) into (2), we get

$$
\begin{array}{r}
\sum_{j_{1}=0}^{N_{1}} \sum_{j_{2}=0}^{N_{2}} \cdots \sum_{j_{m}=0}^{N_{m}} \dot{x}_{j_{1} j_{2} \cdots j_{m}}(t) U_{j_{1}}\left(\xi_{1}\right) U_{j_{2}}\left(\xi_{2}\right) \cdots U_{j_{m}}\left(\xi_{m}\right) \\
=f\left(t, \sum_{j_{1}=0}^{N_{1}} \sum_{j_{2}=0}^{N_{2}} \cdots \sum_{j_{m}=0}^{N_{m}} x_{j_{1} j_{2} \cdots j_{m}}(t) U_{j_{1}}\right. \\
\left.\times\left(\xi_{1}\right) U_{j_{2}}\left(\xi_{2}\right) \cdots U_{j_{m}}\left(\xi_{m}\right)\right) .
\end{array}
$$

According to the results in [26], the orthogonality of polynomials can be expressed as

$$
\int_{a}^{b} \rho(\xi) U_{i}(\xi) U_{j}(\xi) d \xi= \begin{cases}\delta_{j}, & i=j \\ 0, & i \neq j\end{cases}
$$

and the orthogonal polynomials satisfy the following recurrent relation:

$$
\xi U_{j}(\xi)=\alpha_{j-1} U_{j-1}(\xi)+\beta_{j} U_{j}(\xi)+\gamma_{j+1} U_{j+1}(\xi),
$$

where $U_{-1}(\xi)=0, U_{0}(\xi)=1, \alpha_{j-1}=\left(\delta_{j} / \delta_{j-1}\right)\left(a_{j-1} / a_{j}\right), \beta_{j}=$ $\left(1 / \delta_{j}\right) \int_{a}^{b} \xi \rho(\xi) U_{j}^{2}(\xi) d \xi, \gamma_{j+1}=a_{j} / a_{j+1}$, and $a_{j}$ is the coefficient of the item $\xi^{j}$ for polynomial $U_{j}(\xi)$.

Then the quadratic product polynomial of (4) can be simplified into a linear combination of related single polynomials via the recurrent relation. By calculation, we have

$$
\begin{array}{r}
\sum_{j_{1}=0}^{N_{1}} \sum_{j_{2}=0}^{N_{2}} \cdots \sum_{j_{m}=0}^{N_{m}} \dot{x}_{j_{1} j_{2} \cdots j_{m}}(t) U_{j_{1}}\left(\xi_{1}\right) U_{j_{2}}\left(\xi_{2}\right) \cdots U_{j_{m}}\left(\xi_{m}\right) \\
=\tilde{f}\left(t, \sum_{j_{1}=0}^{N_{1}} \sum_{j_{2}=0}^{N_{2}} \cdots \sum_{j_{m}=0}^{N_{m}} x_{j_{1} j_{2} \cdots j_{m}}(t) U_{j_{1}}\right. \\
\left.\times\left(\xi_{1}\right) U_{j_{2}}\left(\xi_{2}\right) \cdots U_{j_{m}}\left(\xi_{m}\right)\right),
\end{array}
$$

where $\tilde{f}(t, x)=\left(\tilde{f}_{1}(t, x), \tilde{f}_{2}(t, x), \ldots, \tilde{f}_{n}(t, x)\right)^{T}$ is a continuous linear vector function. Multiply both sides of (7) by 
$U_{j_{1}}\left(\xi_{1}\right) \cdots U_{j_{m}}\left(\xi_{m}\right)\left(j_{1}=0,1, \ldots, N_{1} ; \ldots ; j_{m}=0,1, \ldots, N_{m}\right)$ and take expectation. Based on the orthogonality of the polynomial approximation, the equivalent deterministic system is

$$
\dot{\tilde{x}}(t, \tilde{x})=\tilde{f}(t, \tilde{x}),
$$

where $\tilde{x}=\left(x_{0101 \cdots 0 m} \cdots x_{N_{1} 1 \cdots N_{m} m}\right)^{T}$. Then dynamical features of the nonlinear chaotic system with multistochastic disturbances can be obtained by analyzing its equivalent deterministic system.

Remark 2. Based on the probability density function $\rho\left(\xi_{i}\right)$ defined on $[a, b]$, appropriate orthogonal polynomial $U\left(\xi_{i}\right)$ is chosen. For example, one has the following.

(i) The second kind of Chebyshev polynomial associated with the arch distribution is defined on $[-1,1]$.

(ii) Legendre polynomial with the uniform distribution is defined on $[-1,1]$.

(iii) Laguerre polynomial with the Gamma distribution is defined on $[0,+\infty)$.

(iv) Hermite polynomial with the Gaussian distribution is defined on $(-\infty,+\infty)$.

(v) Jacobi polynomials with the Beta distribution is defined on $[0,1]$.

Readers are referred to [26] for further information about the relationships among these orthogonal polynomials.

\section{Orthogonal Polynomial Approximation for the Lorenz System with Multistochastic Disturbances}

As a representative of the chaos, the Lorenz-like systems have been analyzed [27-29]. Among them, Chen system and Lü system have been proved to be the special cases of the Lorenz system in $[30,31]$. The Lorenz system has become one of the most widely studied nonlinear chaotic systems because of its wide range of behaviors [32-34]. It has been found to be useful in statistics, engineering science, and so on [35-37]. Therefore, the research on the Lorenz system with multistochastic disturbances has very important theoretical significance. Next, the Lorenz system with multistochastic disturbances will be considered. The complex dynamic behaviors are discussed with performing the numerical simulations of its equivalent deterministic system.

The Lorenz system is

$$
\begin{gathered}
\dot{x}=a(y-x), \\
\dot{y}=c x-x z-y, \\
\dot{z}=x y-b z,
\end{gathered}
$$

where $x, y$, and $z$ are state variables. $a, b$, and $c$ are unknown parameters to be identified. In particular, when $a=10$, $b=8 / 3$, and $c=28$, the system (9) displays a chaotic attractor. In this paper, we consider the Lorenz system with multistochastic disturbances. If $b$ and $c$ are independently random parameters, then (9) becomes the stochastic Lorenz system. Suppose that $b$ and $c$ can be expressed as

$$
\begin{aligned}
& b=\bar{b}+\delta_{1} \xi_{1}, \\
& c=\bar{c}+\delta_{2} \xi_{2},
\end{aligned}
$$

where $\bar{b}$ and $\bar{c}$ are mean values of $b$ and $c$, respectively; $\xi_{1}, \xi_{2}$ are independent identically distributed random variables defined on $[a, b]$ with the probability density function $\rho\left(\xi_{i}\right)(i=1,2) ; \delta_{1}, \delta_{2}\left(\delta_{1}, \delta_{2}>0\right)$ are designed as intensities of the random parameters $b$ and $c$, respectively.

Then, the stochastic Lorenz system can be written as

$$
\begin{gathered}
\dot{x}=a(y-x), \\
\dot{y}=\left(\bar{c}+\delta_{2} \xi_{2}\right) x-x z-y, \\
\dot{z}=x y-\left(\bar{b}+\delta_{1} \xi_{1}\right) z .
\end{gathered}
$$

According to (3), the responses can be written as

$$
\begin{aligned}
& x=x\left(t, \xi_{1}, \xi_{2}\right)=\sum_{i=0}^{M} \sum_{j=0}^{N} x_{i j}(t) U_{i}\left(\xi_{1}\right) U_{j}\left(\xi_{2}\right), \\
& y=y\left(t, \xi_{1}, \xi_{2}\right)=\sum_{i=0}^{M} \sum_{j=0}^{N} y_{i j}(t) U_{i}\left(\xi_{1}\right) U_{j}\left(\xi_{2}\right), \\
& z=z\left(t, \xi_{1}, \xi_{2}\right)=\sum_{i=0}^{M} \sum_{j=0}^{N} z_{i j}(t) U_{i}\left(\xi_{1}\right) U_{j}\left(\xi_{2}\right),
\end{aligned}
$$

where $x_{i j}=\iint_{a}^{b} \rho\left(\xi_{1}\right) \rho\left(\xi_{2}\right) U_{i}\left(\xi_{1}\right) U_{j}\left(\xi_{2}\right) x\left(t, \xi_{1}, \xi_{2}\right) d \xi_{1} d \xi_{2}$, $y_{i j}=\iint_{a}^{b} \rho\left(\xi_{1}\right) \rho\left(\xi_{2}\right) U_{i}\left(\xi_{1}\right) U_{j}\left(\xi_{2}\right) y\left(t, \xi_{1}, \xi_{2}\right) d \xi_{1} d \xi_{2}, z_{i j}=$ $\iint_{a}^{b} \rho\left(\xi_{1}\right) \rho\left(\xi_{2}\right) U_{i}\left(\xi_{1}\right) U_{j}\left(\xi_{2}\right) z\left(t, \xi_{1}, \xi_{2}\right) d \xi_{1} d \xi_{2} . \quad U_{i}\left(\xi_{1}\right), \quad U_{j}\left(\xi_{2}\right)$ represent the $i$ th, $j$ th orthogonal polynomials, respectively, and $M, N$ represent the largest order of the polynomials.

Substituting (12) into (11), we get

$$
\begin{gathered}
\sum_{i=0}^{M} \sum_{j=0}^{N} \dot{x}_{i j}(t) U_{i}\left(\xi_{1}\right) U_{j}\left(\xi_{2}\right) \\
=a\left[\sum_{i=0}^{M} \sum_{j=0}^{N} y_{i j}(t) U_{i}\left(\xi_{1}\right) U_{j}\left(\xi_{2}\right)\right. \\
\left.-\sum_{i=0}^{M} \sum_{j=0}^{N} x_{i j}(t) U_{i}\left(\xi_{1}\right) U_{j}\left(\xi_{2}\right)\right], \\
\sum_{i=0}^{M} \sum_{j=0}^{N} \dot{y}_{i j}(t) U_{i}\left(\xi_{1}\right) U_{j}\left(\xi_{2}\right) \\
=\bar{c} \sum_{i=0}^{M} \sum_{j=0}^{N} x_{i j}(t) U_{i}\left(\xi_{1}\right) U_{j}\left(\xi_{2}\right) \\
-\sum_{i=0}^{M} \sum_{j=0}^{N} y_{i j}(t) U_{i}\left(\xi_{1}\right) U_{j}\left(\xi_{2}\right)
\end{gathered}
$$




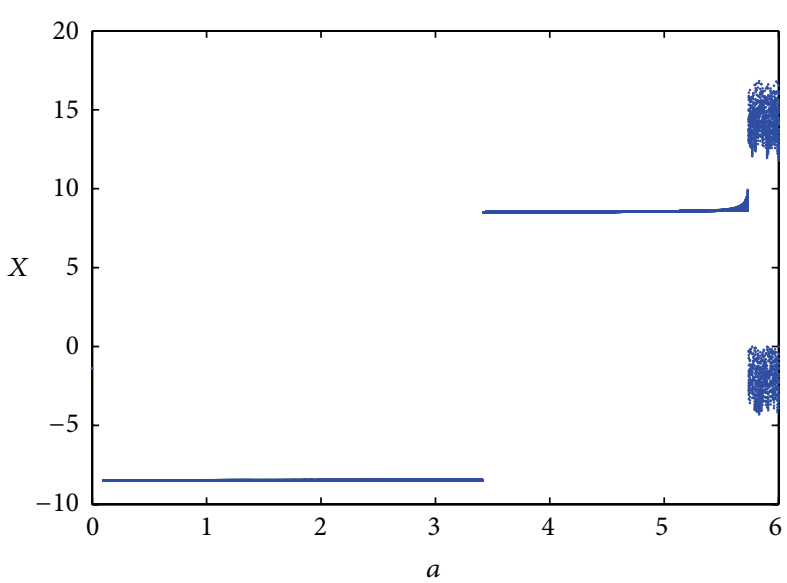

DR

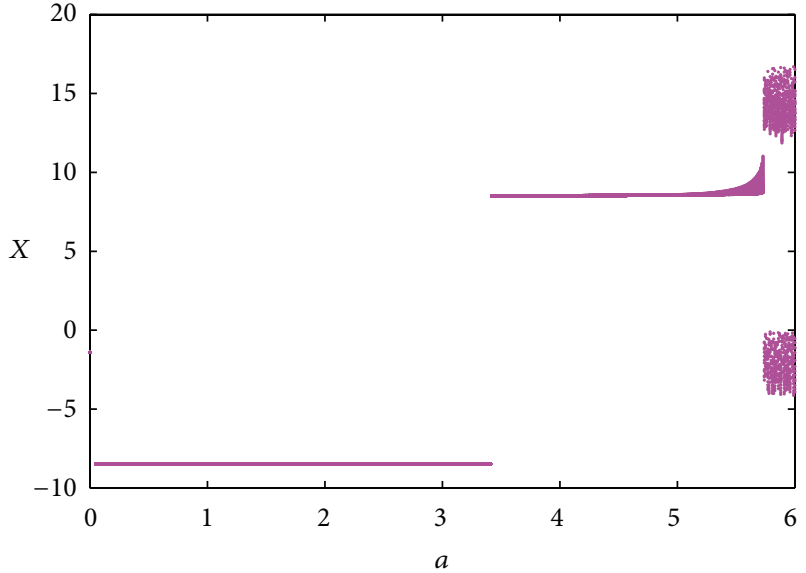

SRM

(a)

(b)

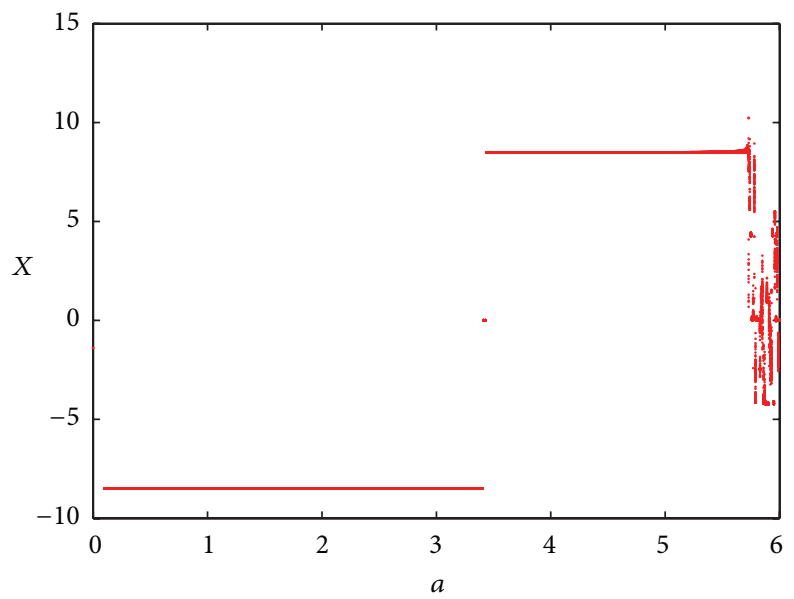

EMR

(c)

FIGURE 1: The bifurcation diagrams of the three responses.

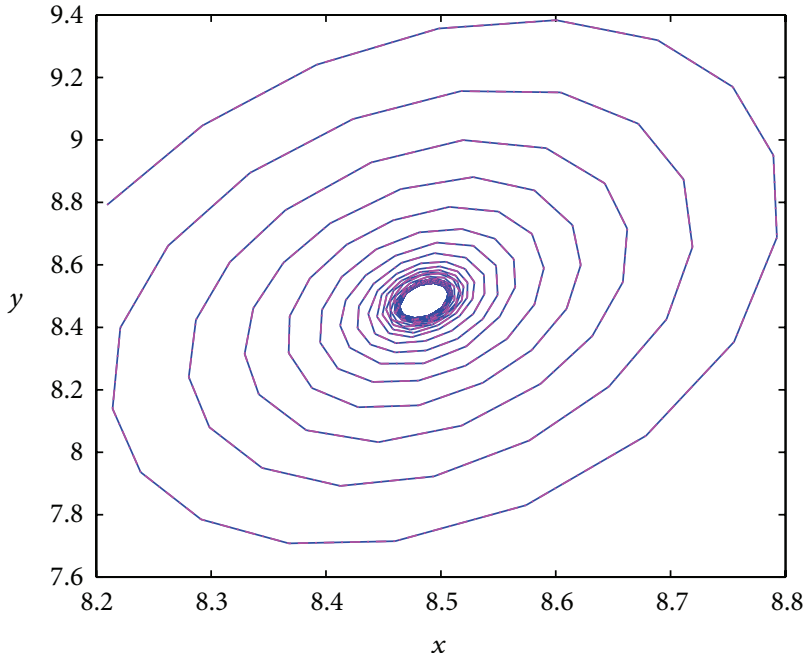

- DR

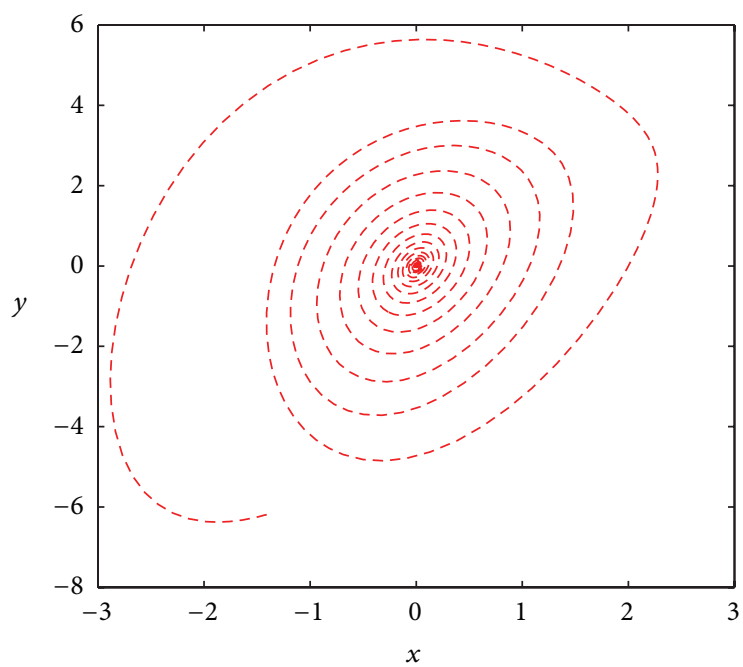

- - EMR

(a)

(b)

FIGURE 2: The phase portraits of the three responses with $a=3.43$. 


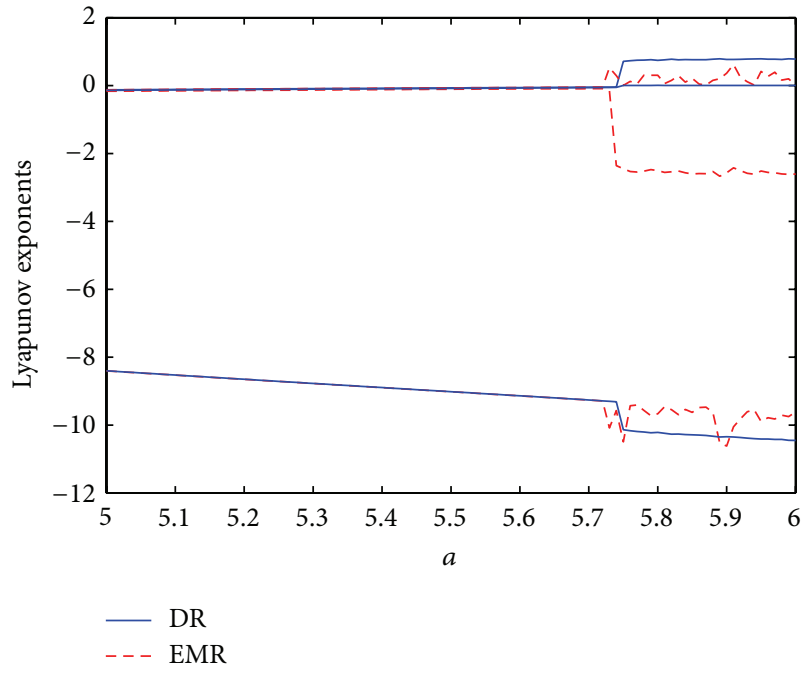

FIGURE 3: The maximum Lyapunov exponents for the two systems with $\delta_{1}=\delta_{2}=0.01$.

$$
\begin{aligned}
&-\left[\sum_{i=0}^{M} \sum_{j=0}^{N} x_{i j}(t) U_{i}\left(\xi_{1}\right) U_{j}\left(\xi_{2}\right)\right] \\
& \times\left[\sum_{i=0}^{M} \sum_{j=0}^{N} z_{i j}(t) U_{i}\left(\xi_{1}\right) U_{j}\left(\xi_{2}\right)\right] \\
&+\delta_{2} \xi_{2} \sum_{i=0}^{M} \sum_{j=0}^{N} x_{i j}(t) U_{i}\left(\xi_{1}\right) U_{j}\left(\xi_{2}\right), \\
& \sum_{i=0}^{M} \sum_{j=0}^{N} \dot{z}_{i j}(t) U_{i}\left(\xi_{1}\right) U_{j}\left(\xi_{2}\right) \\
&=\left[\sum_{i=0}^{M} \sum_{j=0}^{N} x_{i j}(t) U_{i}\left(\xi_{1}\right) U_{j}\left(\xi_{2}\right)\right] \\
& \quad\left[\sum_{i=0}^{M} \sum_{j=0}^{N} y_{i j}(t) U_{i}\left(\xi_{1}\right) U_{j}\left(\xi_{2}\right)\right] \\
& \quad-\bar{b} \sum_{i=0}^{M} \sum_{j=0}^{N} z_{i j}(t) U_{i}\left(\xi_{1}\right) U_{j}\left(\xi_{2}\right) \\
&-\delta_{1} \xi_{1} \sum_{i=0}^{M} \sum_{j=0}^{N} z_{i j}(t) U_{i}\left(\xi_{1}\right) U_{j}\left(\xi_{2}\right) .
\end{aligned}
$$

Similar to [23], we choose the random variables $\xi_{i}$ ( $i=$ $1,2)$ defined on $[-1,1]$ with an arch-like distribution in the rest of the paper. The arch-like probability density function

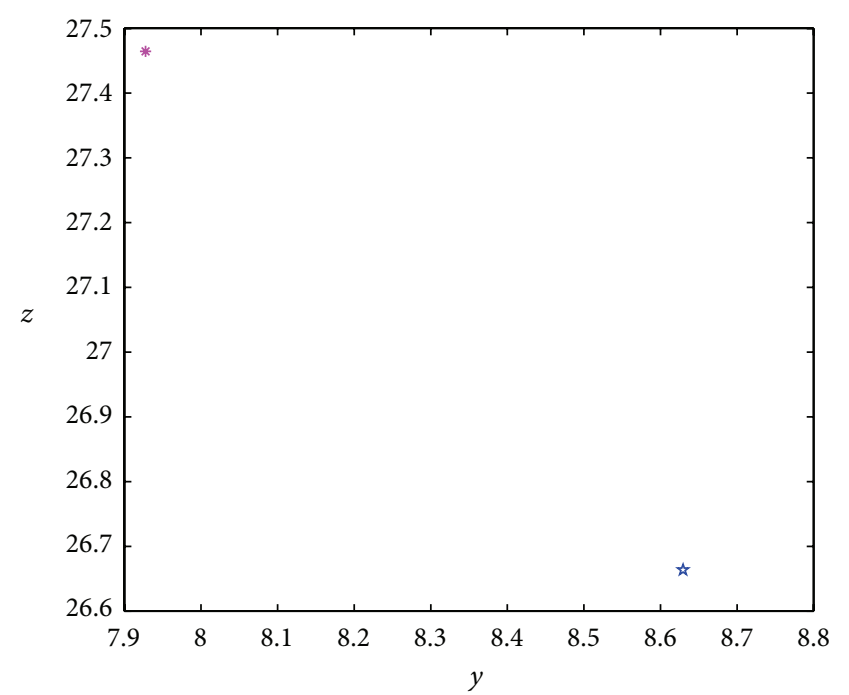

* $\mathrm{DR}$

* SRM

(a)

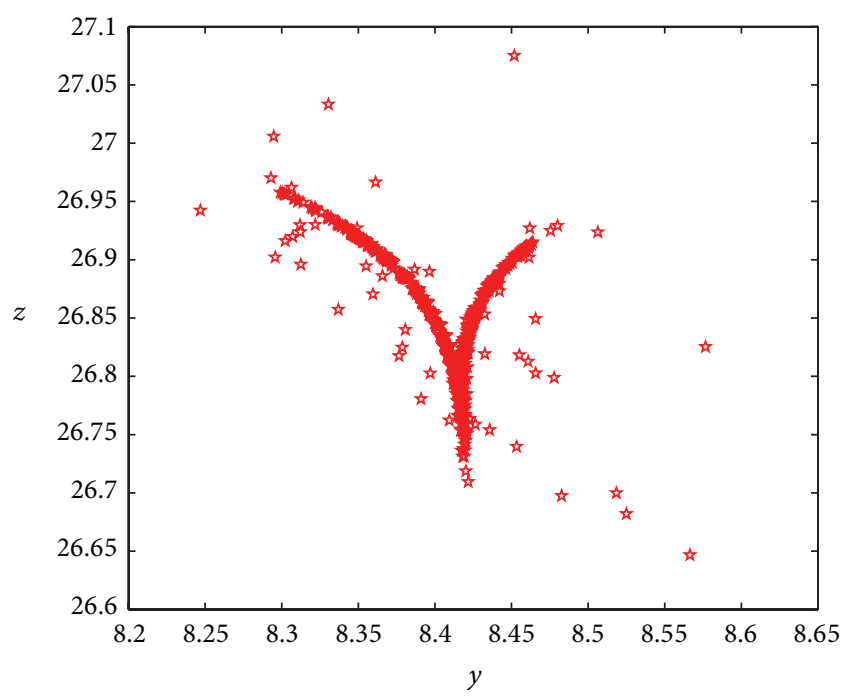

\# EMR

(b)

FIGURE 4: The Poincaré sections of the three responses with $a=5.73$.

$\rho(\xi)$ is usually characterized by the uncertainty in the real word; that is,

$$
\rho(\xi)= \begin{cases}\left(\frac{2}{\pi}\right) \sqrt{1-\xi^{2}}, & |\xi| \leq 1, \\ 0, & |\xi|>1 .\end{cases}
$$

Corresponding to these random variables, the orthogonal polynomial is chosen as the second kind of Chebyshev polynomial. And the orthogonality of the polynomial can be expressed as

$$
\int_{-1}^{1}\left(\frac{2}{\pi \sqrt{1-\xi^{2}}}\right) U_{i}(\xi) U_{j}(\xi) d \xi= \begin{cases}1, & i=j \\ 0, & i \neq j\end{cases}
$$




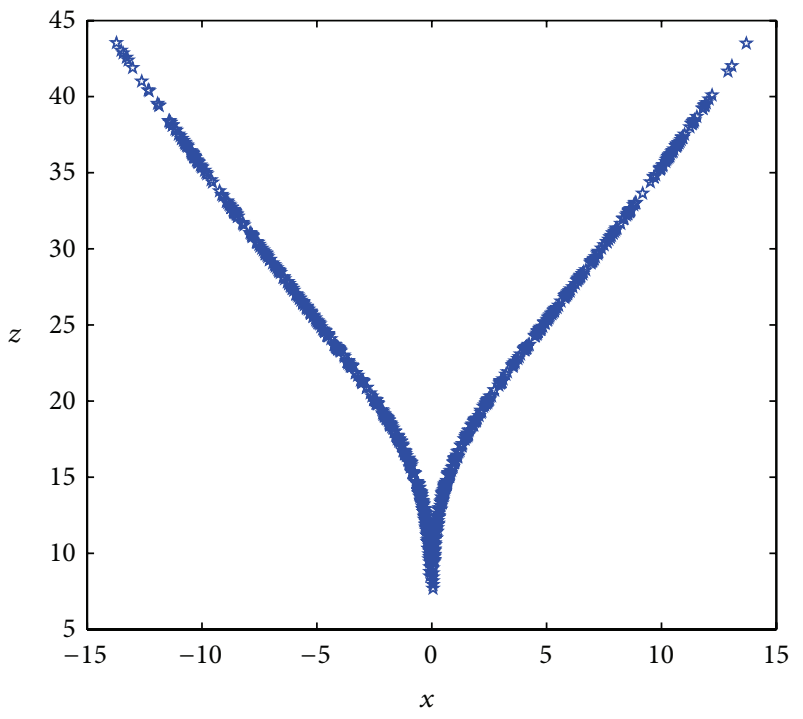

\& $\mathrm{DR}$

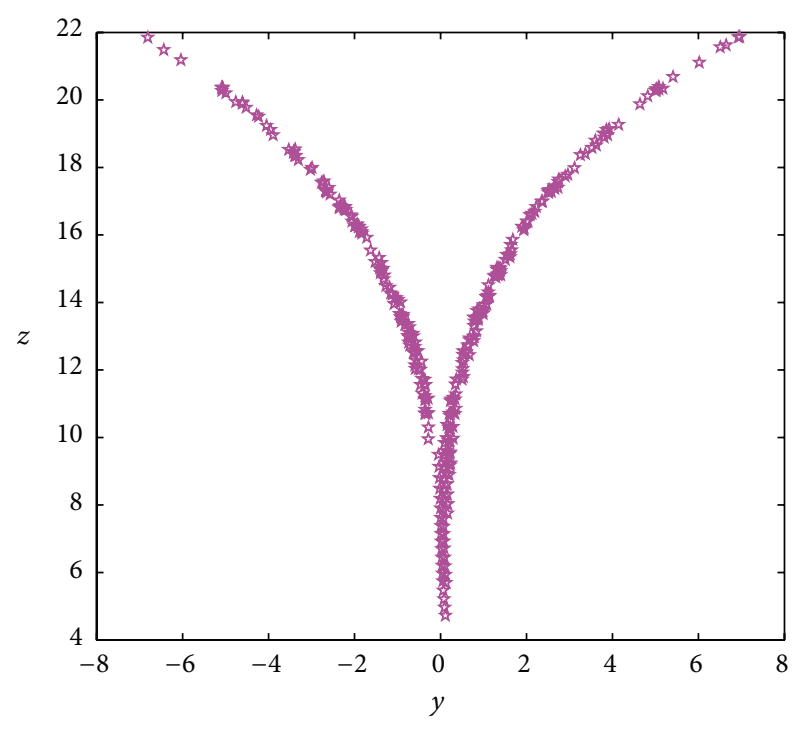

\& SRM

(a)

(b)

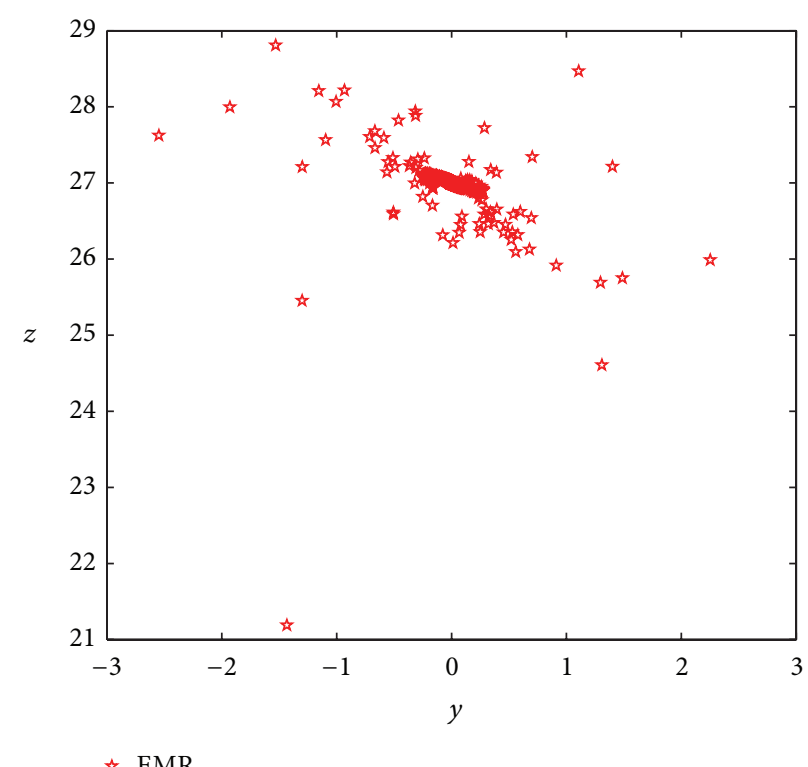

(c)

FIgURe 5: The Poincaré sections of the three responses with $a=5.91$.

Therefore, the coefficients of (6) are $\alpha_{j-1}=\gamma_{j+1}=1 / 2$ and $\beta_{j}=0(j=0,1,2, \ldots)$. Furthermore, the quadratic product polynomial of (13) can be simplified into a linear combination of the related single polynomials via the recurrent relation. The coefficients of $U_{i}\left(\xi_{1}\right) U_{j}\left(\xi_{2}\right)$ can be denoted as $X Y_{i j}(t)$ or $X Z_{i j}(t)$ :

$$
\begin{aligned}
& {\left[\sum_{i=0}^{M} \sum_{j=0}^{N} x_{i j}(t) U_{i}\left(\xi_{1}\right) U_{j}\left(\xi_{2}\right)\right]} \\
& \times\left[\sum_{i=0}^{M} \sum_{j=0}^{N} z_{i j}(t) U_{i}\left(\xi_{1}\right) U_{j}\left(\xi_{2}\right)\right]
\end{aligned}
$$

$$
\begin{gathered}
=\sum_{i=0}^{M} \sum_{j=0}^{N} X Z_{i j}(t) U_{i}\left(\xi_{1}\right) U_{j}\left(\xi_{2}\right), \\
{\left[\sum_{i=0}^{M} \sum_{j=0}^{N} x_{i j}(t) U_{i}\left(\xi_{1}\right) U_{j}\left(\xi_{2}\right)\right]} \\
\times\left[\sum_{i=0}^{M} \sum_{j=0}^{N} y_{i j}(t) U_{i}\left(\xi_{1}\right) U_{j}\left(\xi_{2}\right)\right] \\
=\sum_{i=0}^{M} \sum_{j=0}^{N} X Y_{i j}(t) U_{i}\left(\xi_{1}\right) U_{j}\left(\xi_{2}\right),
\end{gathered}
$$




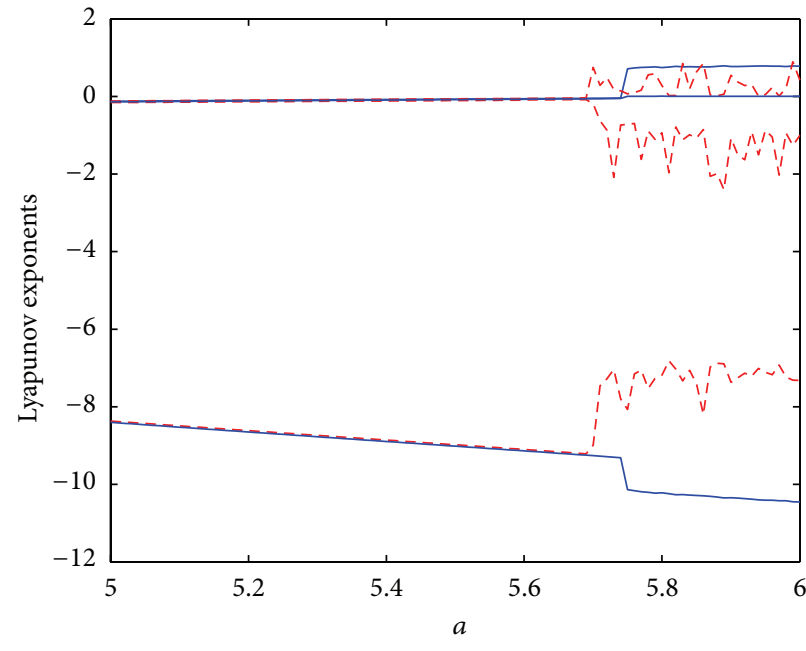

$\begin{array}{ll}- & \text { DR } \\ --- & \text { EMR }\end{array}$

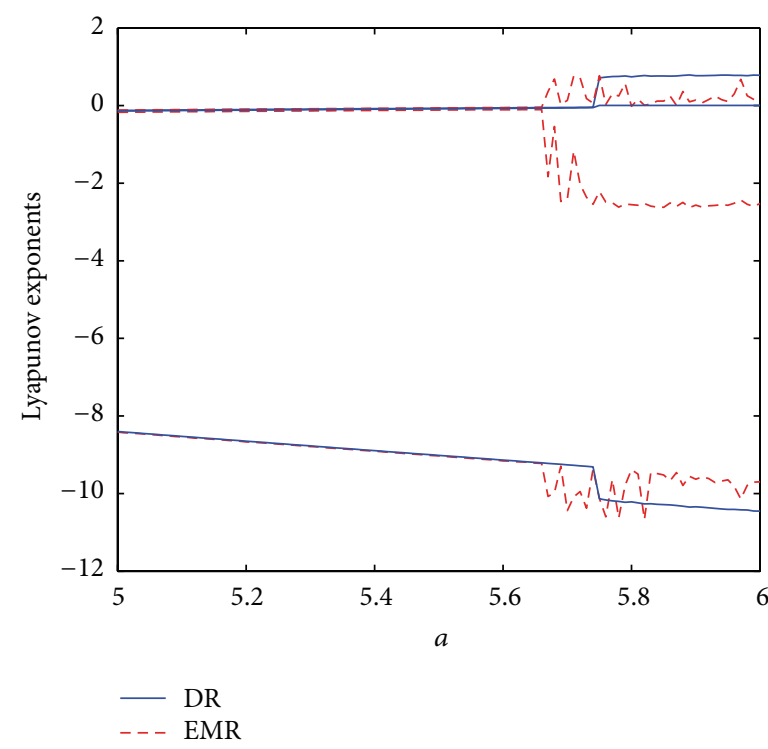

(b) $\delta_{1}=0.05, \delta_{2}=0.01$

(a) $\delta_{1}=0.03, \delta_{2}=0.01$

FIGURE 6: The maximum Lyapunov exponents for the two systems.

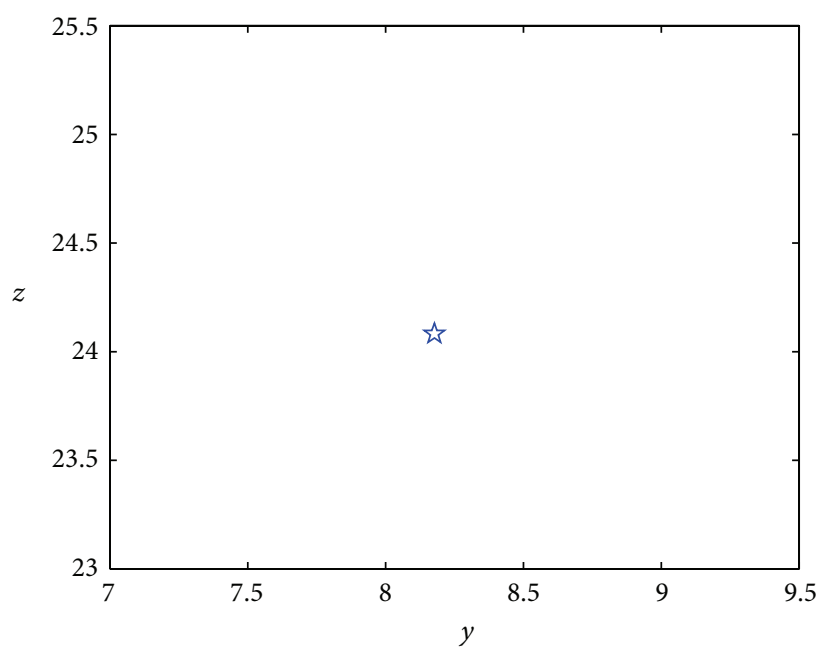

is $\mathrm{DR}$

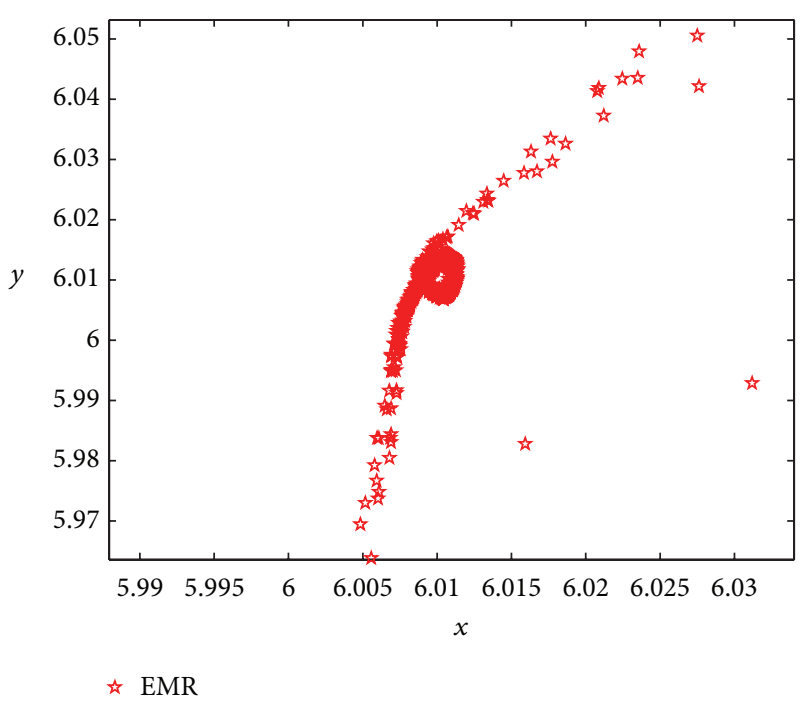

(b)

(a)

FIgURe 7: The Poincaré sections of the two systems with $a=5.66$.

where $X Y_{i j}(t)$ or $X Z_{i j}(t)(i=0,1, \ldots, M ; j=0,1, \ldots, N)$ can be derived through the computer algebraic system. Substituting (6) and (16) into (13), we yield

$$
\begin{aligned}
& \sum_{i=0}^{M} \sum_{j=0}^{N} \dot{x}_{i j}(t) U_{i}\left(\xi_{1}\right) U_{j}\left(\xi_{2}\right) \\
&=a\left[\sum_{i=0}^{M} \sum_{j=0}^{N} y_{i j}(t) U_{i}\left(\xi_{1}\right) U_{j}\left(\xi_{2}\right)\right. \\
&\left.\quad-\sum_{i=0}^{M} \sum_{j=0}^{N} x_{i j}(t) U_{i}\left(\xi_{1}\right) U_{j}\left(\xi_{2}\right)\right],
\end{aligned}
$$

$$
\begin{aligned}
\sum_{i=0}^{M} \sum_{j=0}^{N} \dot{y}_{i j}(t) U_{i}\left(\xi_{1}\right) U_{j}\left(\xi_{2}\right) \\
=\bar{c} \sum_{i=0}^{M} \sum_{j=0}^{N} x_{i j}(t) U_{i}\left(\xi_{1}\right) U_{j}\left(\xi_{2}\right) \\
\quad-\sum_{i=0}^{M} \sum_{j=0}^{N} y_{i j}(t) U_{i}\left(\xi_{1}\right) U_{j}\left(\xi_{2}\right) \\
\quad-\sum_{i=0}^{M} \sum_{j=0}^{N} X Z_{i j}(t) U_{i}\left(\xi_{1}\right) U_{j}\left(\xi_{2}\right) \\
\quad+\frac{\delta_{2}}{2} \sum_{i=0}^{M} \sum_{j=0}^{N}\left[x_{i j-1}(t)+x_{i j+1}(t)\right] U_{i}\left(\xi_{1}\right) U_{j}\left(\xi_{2}\right),
\end{aligned}
$$




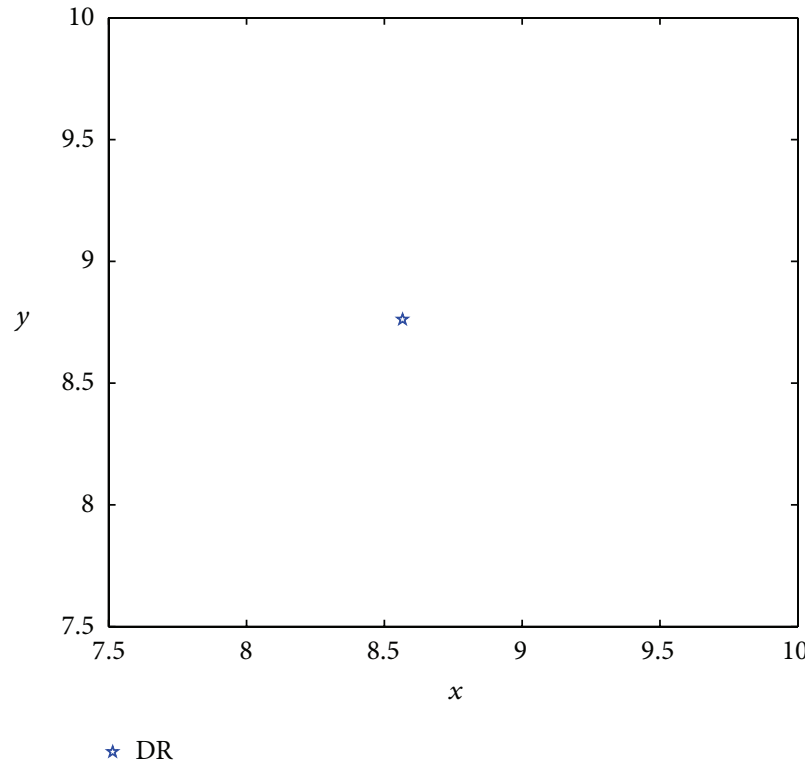

(a)

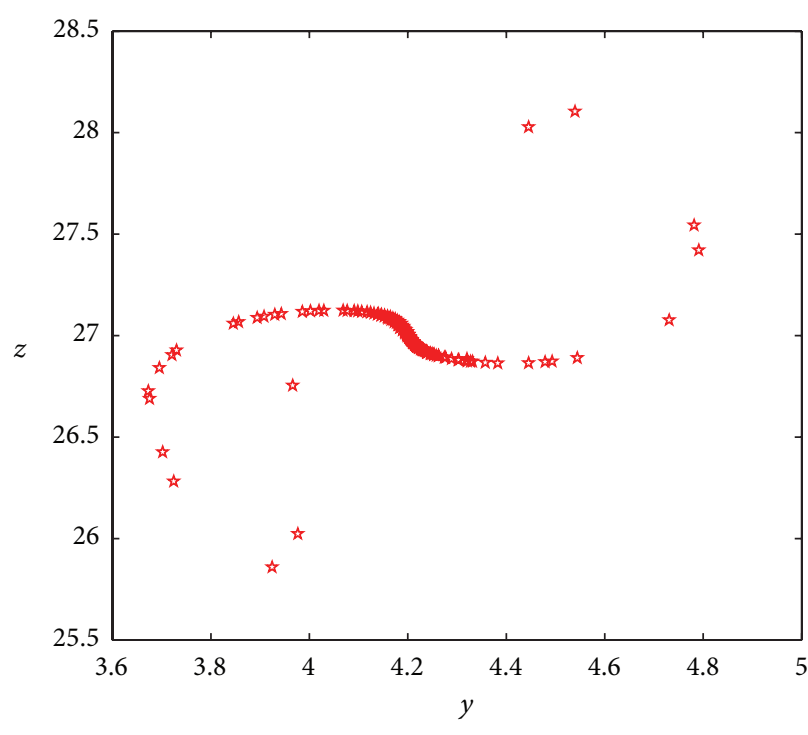

\ $\mathrm{EMR}$

FIgUre 8: The Poincaré sections of the two systems with $a=5.61$.

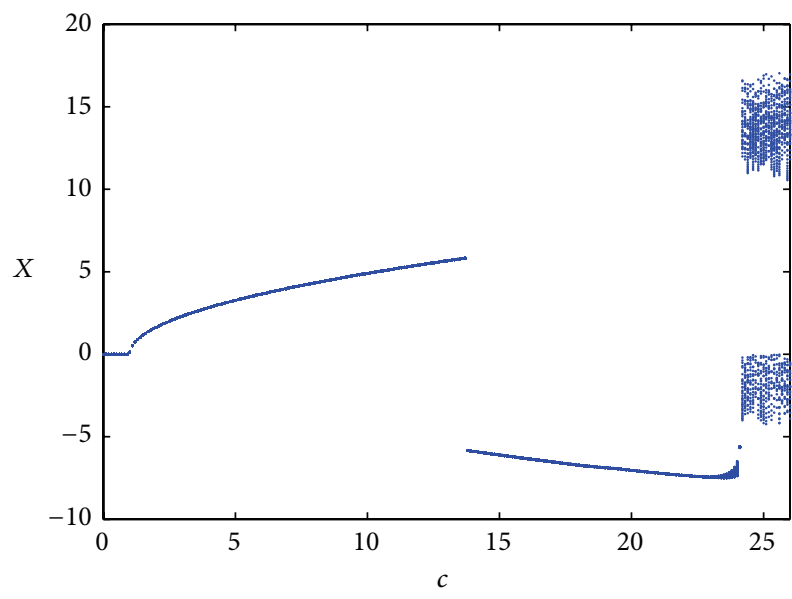

DR

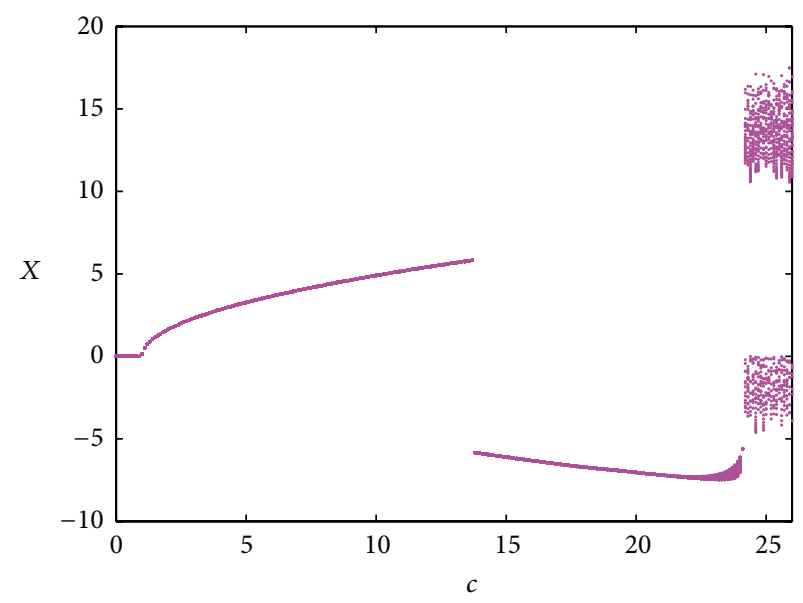

. SRM

(a)

(b)

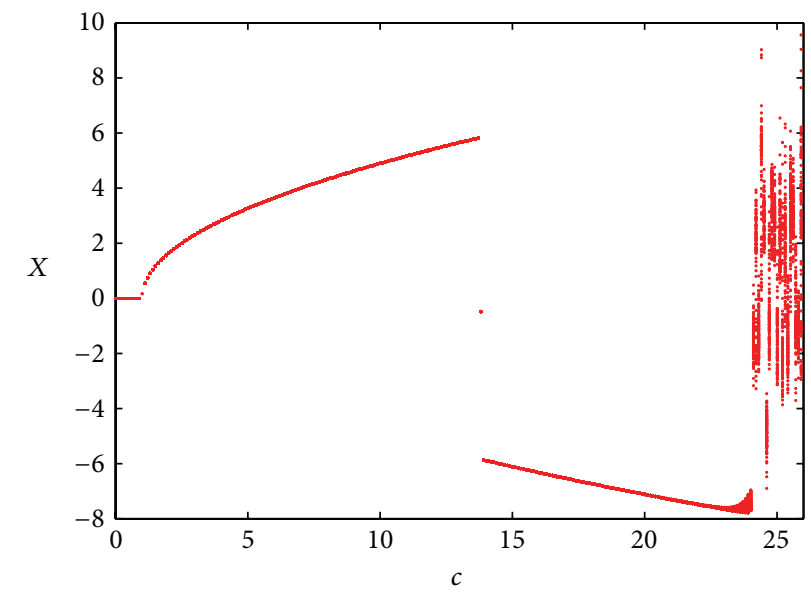

EMR

(c)

FIgURE 9: The bifurcation diagrams of the three responses. 


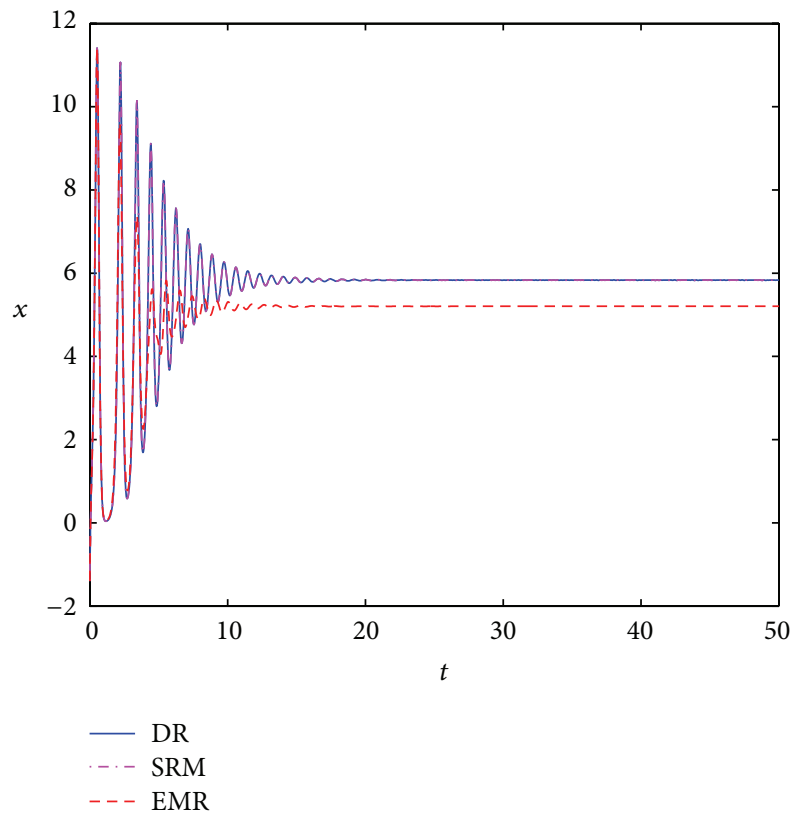

FIGURE 10: The time history of the three responses with $c=13.76$.

$$
\begin{aligned}
& \sum_{i=0}^{M} \sum_{j=0}^{N} \dot{z}_{i j}(t) U_{i}\left(\xi_{1}\right) U_{j}\left(\xi_{2}\right) \\
& =\sum_{i=0}^{M} \sum_{j=0}^{N} X Y_{i j}(t) U_{i}\left(\xi_{1}\right) U_{j}\left(\xi_{2}\right) \\
& \quad-\bar{b} \sum_{i=0}^{M} \sum_{j=0}^{N} z_{i j}(t) U_{i}\left(\xi_{1}\right) U_{j}\left(\xi_{2}\right) \\
& \quad-\frac{\delta_{1}}{2} \sum_{i=0}^{M} \sum_{j=0}^{N}\left[z_{i-1 j}(t)+z_{i+1 j}(t)\right] U_{i}\left(\xi_{1}\right) U_{j}\left(\xi_{2}\right)
\end{aligned}
$$

Based on the result of (8), an equivalent deterministic system of the stochastic Lorenz system (11) is finally obtained when $M=N=3$; that is,

$$
\begin{gathered}
\dot{x}_{i j}(t)=a\left[y_{i j}(t)-x_{i j}(t)\right], \\
\dot{y}_{i j}(t)=\bar{c} x_{i j}(t)-X Z_{i j}(t)-y_{i j}(t) \\
+\frac{\delta_{2}}{2}\left[x_{i j-1}(t)+x_{i j+1}(t)\right], \\
\dot{z}_{i j}(t)=X Y_{i j}(t)-\bar{b} z_{i j}(t)-\frac{\delta_{1}}{2}\left[z_{i-1 j}(t)+z_{i+1 j}(t)\right],
\end{gathered}
$$

where $x_{i,-1}(t)=0, z_{-1, j}(t)=0, x_{i, 4}(t)=0, z_{4, j}(t)=0$, and $(i, j=0,1,2,3)$.
The ensemble mean responses of the system (11) are

$$
\begin{aligned}
& E\left[x\left(t, \xi_{1}, \xi_{2}\right)\right]=\sum_{i=0}^{3} \sum_{j=0}^{3} E\left[x_{i j}(t) U_{i}\left(\xi_{1}\right) U_{j}\left(\xi_{2}\right)\right]=x_{00}(t), \\
& E\left[y\left(t, \xi_{1}, \xi_{2}\right)\right]=\sum_{i=0}^{3} \sum_{j=0}^{3} E\left[y_{i j}(t) U_{i}\left(\xi_{1}\right) U_{j}\left(\xi_{2}\right)\right]=y_{00}(t), \\
& E\left[z\left(t, \xi_{1}, \xi_{2}\right)\right]=\sum_{i=0}^{3} \sum_{j=0}^{3} E\left[z_{i j}(t) U_{i}\left(\xi_{1}\right) U_{j}\left(\xi_{2}\right)\right]=z_{00}(t) .
\end{aligned}
$$

The sample responses can be obtained as follows:

$$
\begin{aligned}
x(t, 0,0) & \approx \sum_{i=0}^{3} \sum_{j=0}^{3} x_{i j}(t) U_{i}(0) U_{j}(0) \\
& =x_{00}(t)-x_{02}(t)-x_{20}(t)+x_{22}(t), \\
y(t, 0,0) & \approx \sum_{i=0}^{3} \sum_{j=0}^{3} y_{i j}(t) U_{i}(0) U_{j}(0) \\
& =y_{00}(t)-y_{02}(t)-y_{20}(t)+y_{22}(t), \\
z(t, 0,0) & \approx \sum_{i=0}^{3} \sum_{j=0}^{3} z_{i j}(t) U_{i}(0) U_{j}(0) \\
& =z_{00}(t)-z_{02}(t)-z_{20}(t)+z_{22}(t) .
\end{aligned}
$$

Given a series of specific initial conditions, the responses of the equivalent deterministic system (18) and the deterministic system (9) can be obtained by numerical simulation. Then the ensemble mean responses (19) and the sample responses (20) are received. Set DR for the deterministic responses of the system (9), set EMR for the ensemble mean responses (19), and set SRM for the sample responses (20). EMR can be used to explore basic nonlinear phenomena in the stochastic Lorenz system (11). The feasibility of the second kind of Chebyshev polynomial approximation is verified by comparing DR with SRM. Some differences of the dynamic behaviors in the stochastic Lorenz system (11) are discussed by comparing DR and EMR.

\section{The Dynamic Analysis}

In this section, dynamic analysis of the three responses DR, SRM, and EMR is explored by MATLAB programming and ode 45 simulating. Owing to the small quantities of intensities $\delta_{1}$ and $\delta_{2}$, the same initial conditions for system (9) and system (18) are given; namely, $x_{0}=x(0)=-1.4, y_{0}=y(0)=$ 2.4, and $z_{0}=z(0)=0$. Consider

$$
\begin{aligned}
& x(0)=[-1.4,0,0,0,0,0,0,0,0,0,0,0,0,0,0,0]^{T}, \\
& y(0)=[2.4,0,0,0,0,0,0,0,0,0,0,0,0,0,0,0]^{T}, \\
& z(0)=[0,0,0,0,0,0,0,0,0,0,0,0,0,0,0,0]^{T} .
\end{aligned}
$$




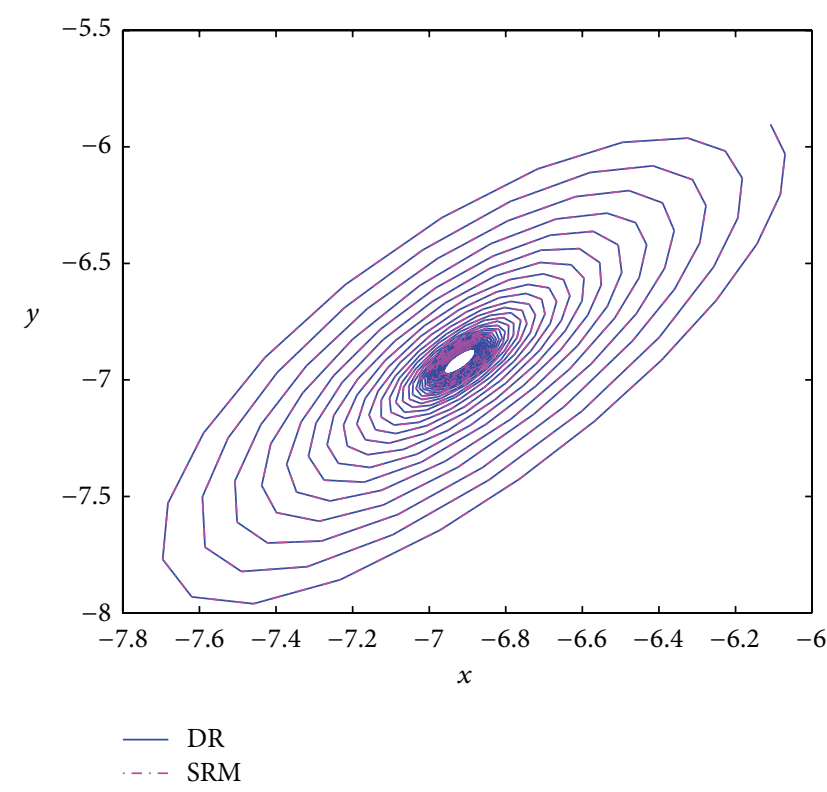

(a)

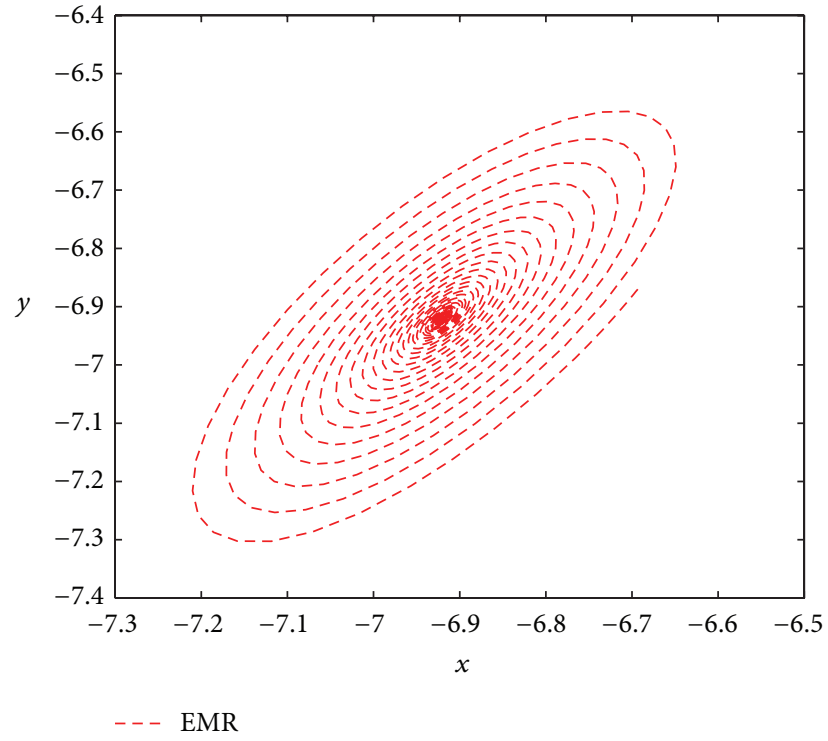

(b)

FIGURE 11: The phase portraits of the three responses with $c=18.96$.

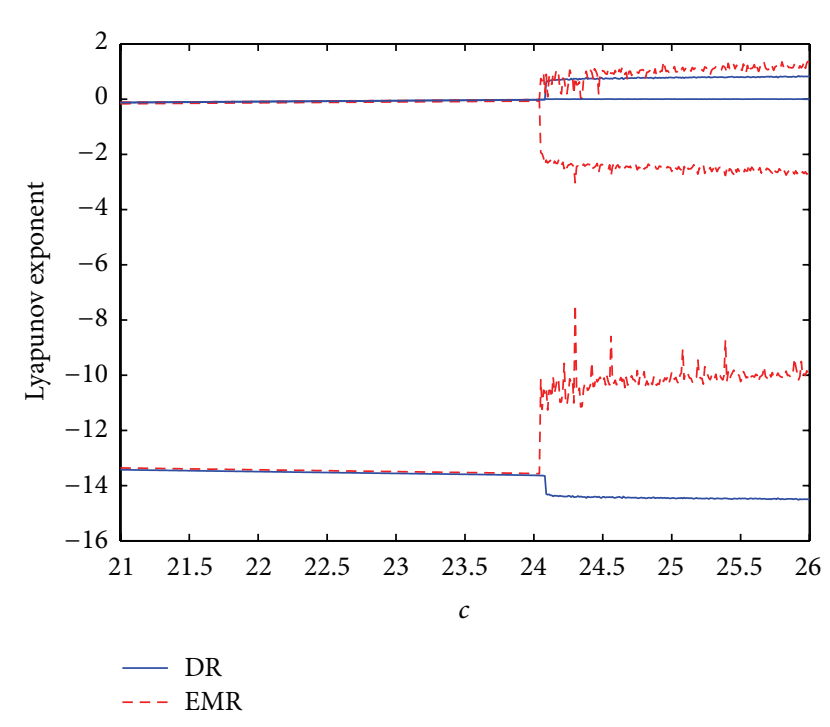

FIGURE 12: The maximum Lyapunov exponents for the two systems with $\delta_{1}=\delta_{2}=0.01$.

It is well known that the dynamic behaviors of the deterministic Lorenz system are complex. With the changing of parameters, the dynamic behaviors of the stochastic Lorenz system (11) are determined by the system parameters and the random parameter disturbances and their intensities. In the following, we analyze the dynamic features of the system (11) in detail.

4.1. Dynamic Analysis of the Stochastic Lorenz System (11) with the Change of System Parameter $a$ and Stochastic Intensity. The system parameters are chosen as $\bar{b}=8 / 3, \bar{c}=28$,
TABLE 1

\begin{tabular}{lcccc}
\hline$a$ & {$[0,0.3]$} & $(0.3,5.72]$ & 5.73 & {$[5.74,6]$} \\
\hline DR & EP & LC & LC & Chaos \\
SRM & EP & LC & LC & Chaos \\
EMR & EP & EP & Chaos & Chaos \\
\hline
\end{tabular}

$\delta_{1}=0.01$, and $\delta_{2}=0.01$ and the initial condition is given as (21). When the parameter $a$ belongs to different intervals, Table 1 shows different dynamic behaviors of the three responses, where EP is the abbreviation of equilibrium point and LC is the abbreviation of limit cycle.

The bifurcation diagrams of the three responses DR, SRM, and EMR are given in Figures 1(a), 1(b), and 1(c). It is found that the trajectories of the three responses DR, SRM, and EMR converge to the same equilibrium point as $a \in[0,0.3]$. DR and SRM converge to the same limit cycle, while EMR still converges to equilibrium point in the interval $(0.3,5.72]$ shown in Figures 2(a) and 2(b). In order to study the chaos phenomenon of the stochastic Lorenz system (11), we discuss the maximum Lyapunov exponent and the Poincaré section. It is clear that EMR enters chaos earlier than DR as revealed in Figure 3. For example, DR and SRM still converge to limit cycle with $a=5.73$ in Figure 4(a). But EMR enters chaos in Figure 4(b). The three responses DR, SRM, and EMR are all chaotic in the interval $[5.74,6]$ such as $a=5.91$ exhibited in Figures 5(a), 5(b), and 5(c).

Increase the stochastic intensity $\delta_{1}$ to 0.03 . From the maximum Lyapunov exponents for the systems (9) and (11) in Figure 6(a), we can find that the stochastic Lorenz system (11) has been chaotic with $a=5.70$. Continue increasing it to 0.05; from the maximum Lyapunov exponents for the systems (9) and (11) in Figure 6(b), we can find that the system (11) has 


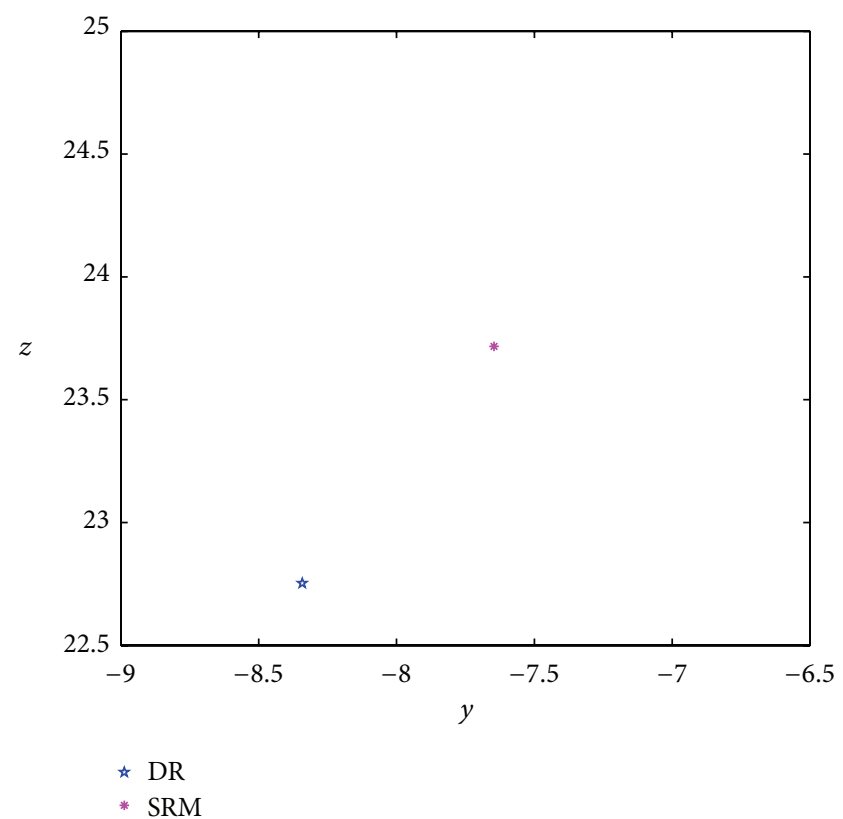

(a)

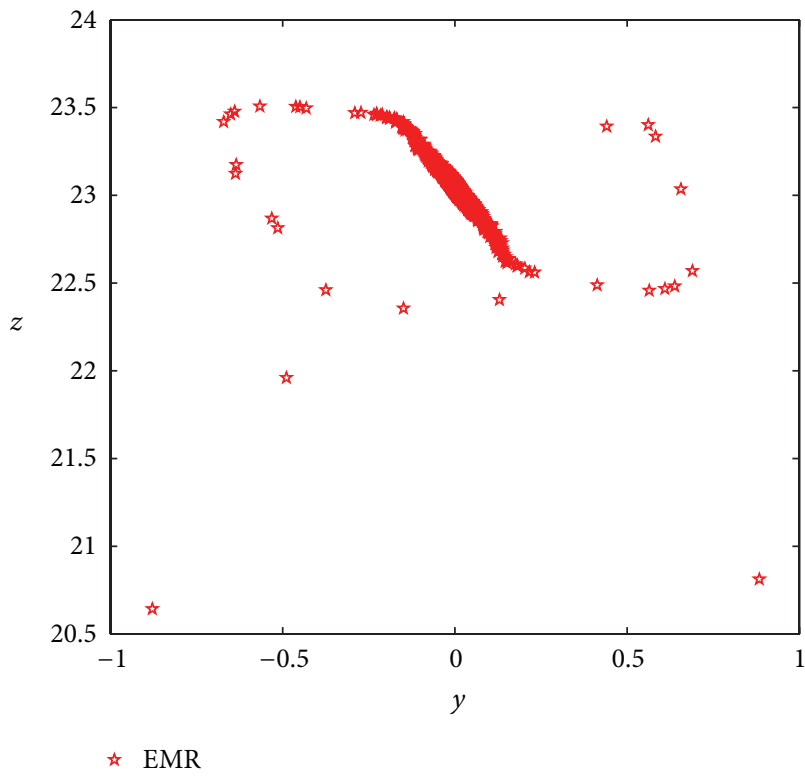

(b)

FIgUre 13: The Poincaré sections of the three responses with $c=24.05$.

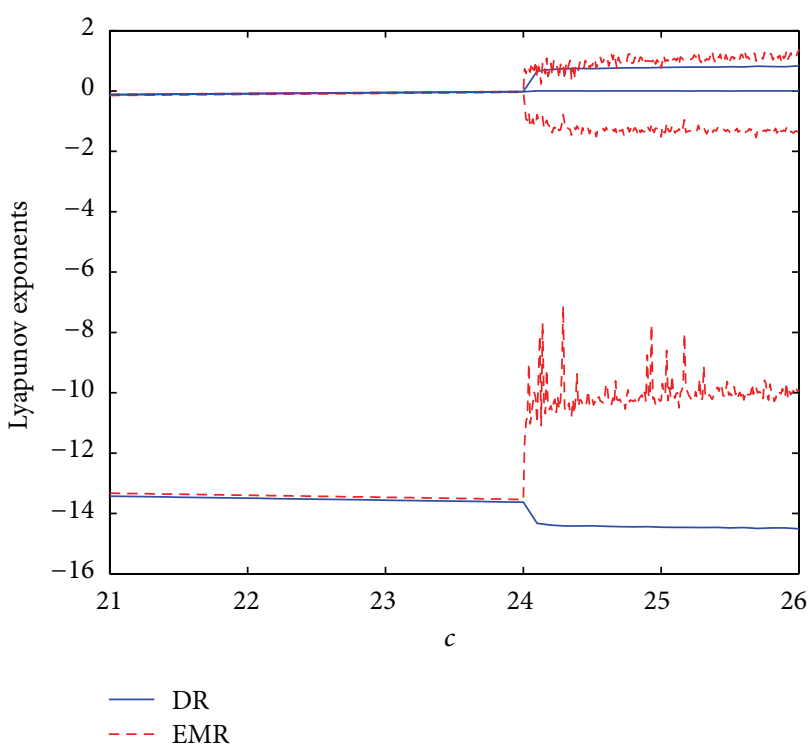

(a) $\delta_{1}=0.03, \delta_{2}=0.01$

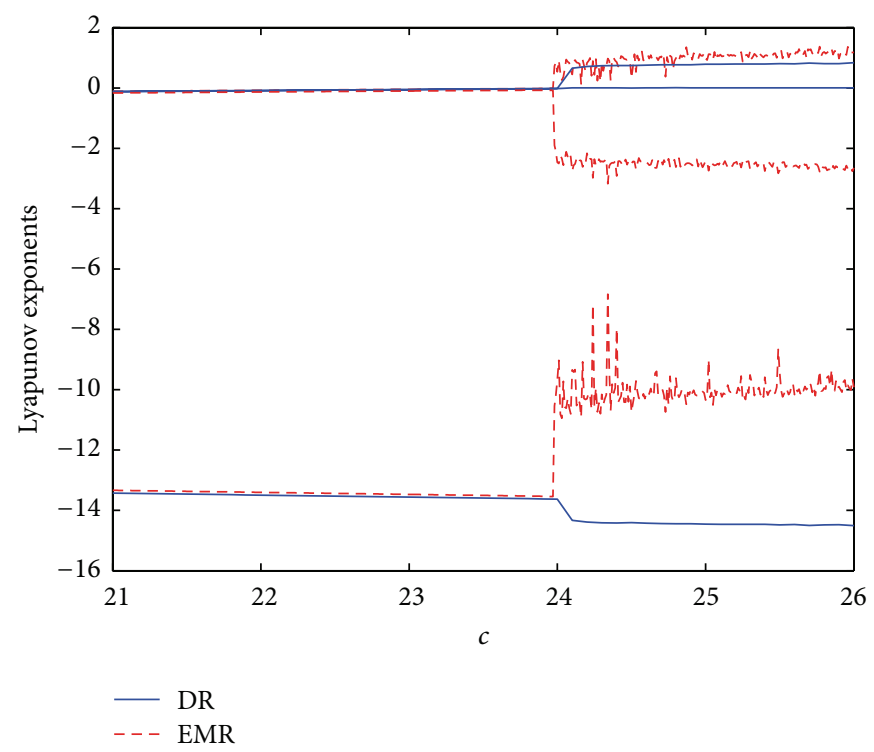

(b) $\delta_{1}=0.05, \delta_{2}=0.01$

FIgURE 14: The maximum Lyapunov exponents for the two systems.

entered chaos with $a=5.68$. Further increase it to 0.08 . The system (9) still converges to limit cycle with $a=5.66$, whereas the system (11) is chaotic, which are, respectively, revealed in Figures $7(\mathrm{a})$ and $7(\mathrm{~b})$. When $\delta_{1}=0.1$, the system (11) is chaotic with $a=5.61$, but the system (9) is not, which are, respectively, revealed in Figures 8(a) and 8(b).

Influenced by the system parameter $a$, EMR does not experience period state before entering chaos. EMR and DR enter chaos at different time periods. With the increase of the stochastic intensity $\delta_{1}$, the stochastic Lorenz system (11) enters chaos earlier than the deterministic system (9).

4.2. Dynamic Analysis of the Stochastic Lorenz System (11) with the Change of Random Parameter $c$ and Stochastic Intensity. Now the system parameters are $a=10, \bar{b}=8 / 3, \delta_{1}=0.01$, and $\delta_{2}=0.01$. The dynamical characteristics of the three responses DR, SRM, and EMR are discussed in Table 2. 
TABle 2

\begin{tabular}{lccccc}
\hline$c$ & {$[0,17.76)$ except for $[13.76,13.85]$} & {$[13.76,13.85]$} & {$[17.76,24.04]$} & {$[24.05,24.10]$} & LC \\
\hline DR & EP & EP & LC & Chaos \\
SRM & EP & EP & LC & LC & Chaos \\
EMR & EP & EP & EP & Chaos & Chaos \\
\hline
\end{tabular}

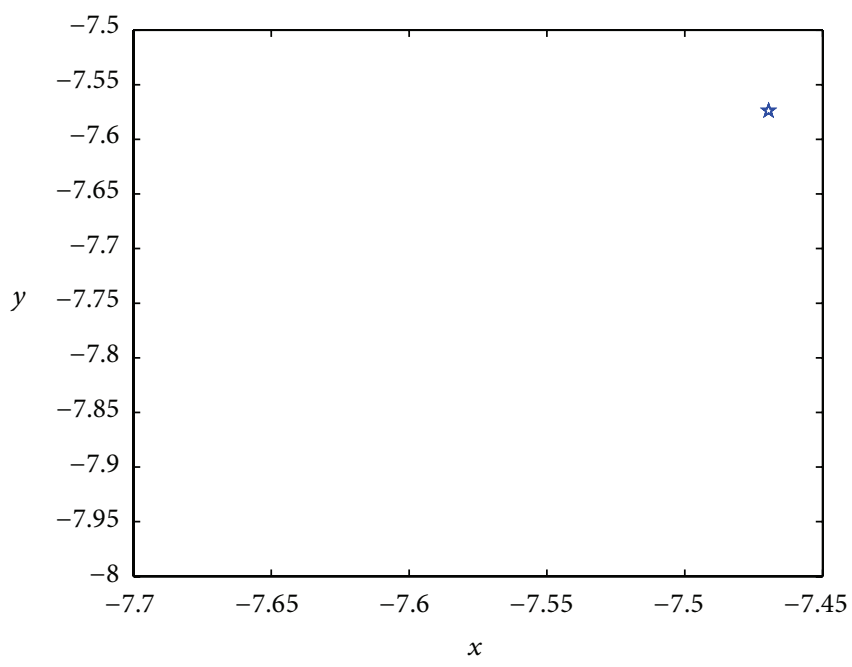

4 $\mathrm{DR}$

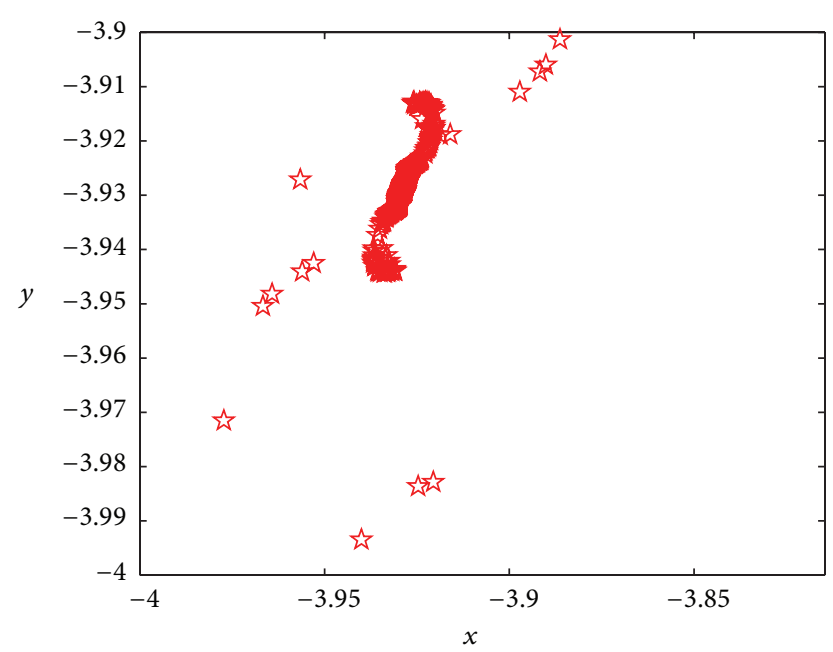

㶦 $\mathrm{EMR}$

(a)

(b)

Figure 15: The Poincare sections of the two systems with $c=23.90$.

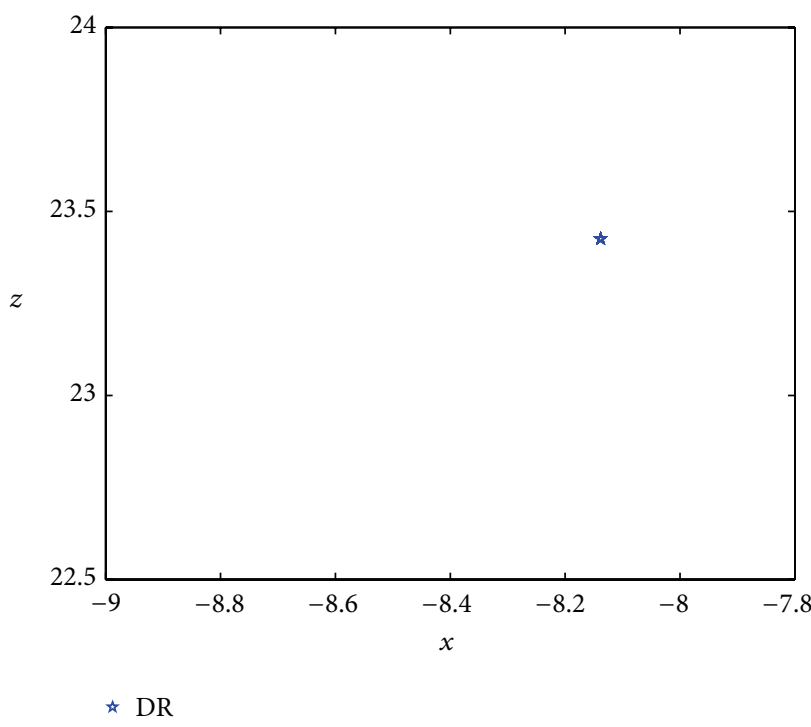

(a)

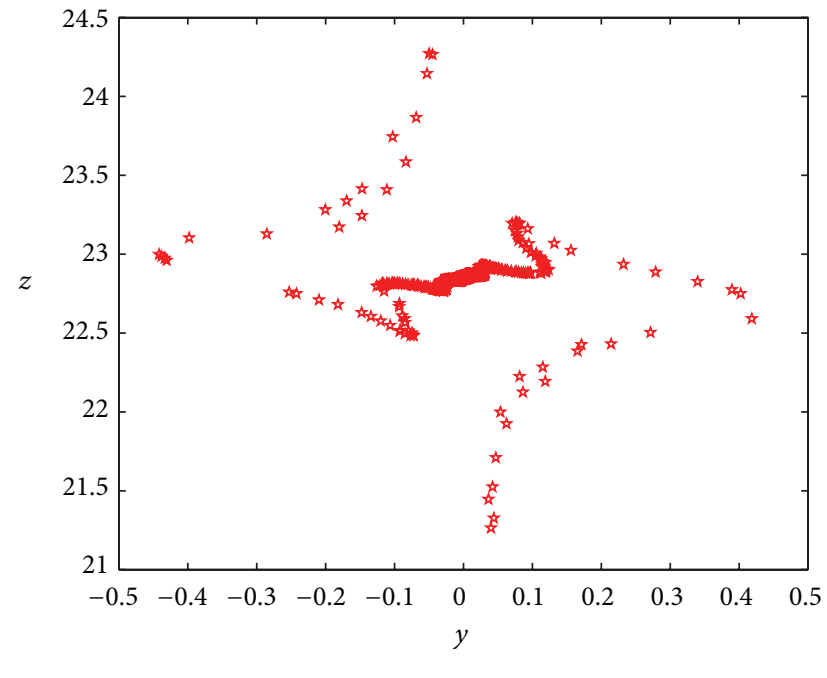

* EMR

Figure 16: The Poincaré sections of the two systems with $c=23.85$. 


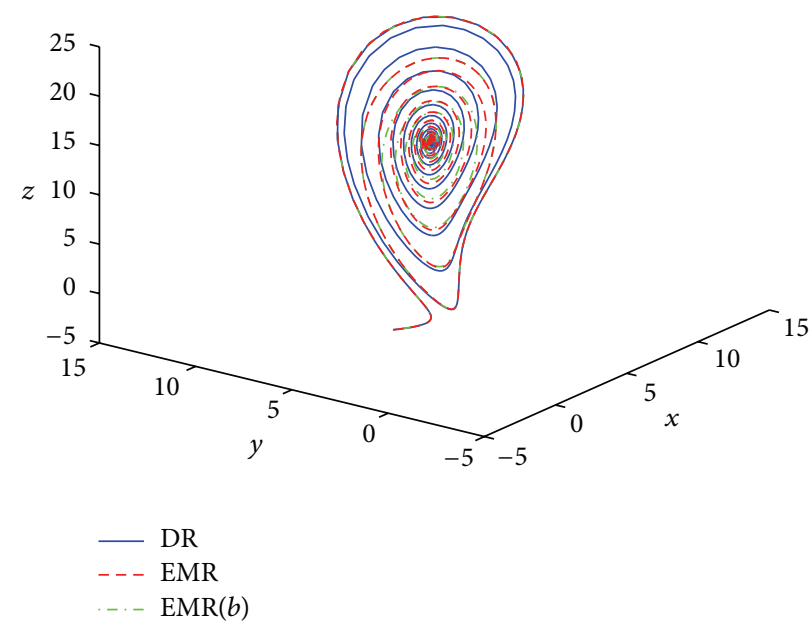

FIGURE 17: The phase portraits of the four responses with $c=13.75$.

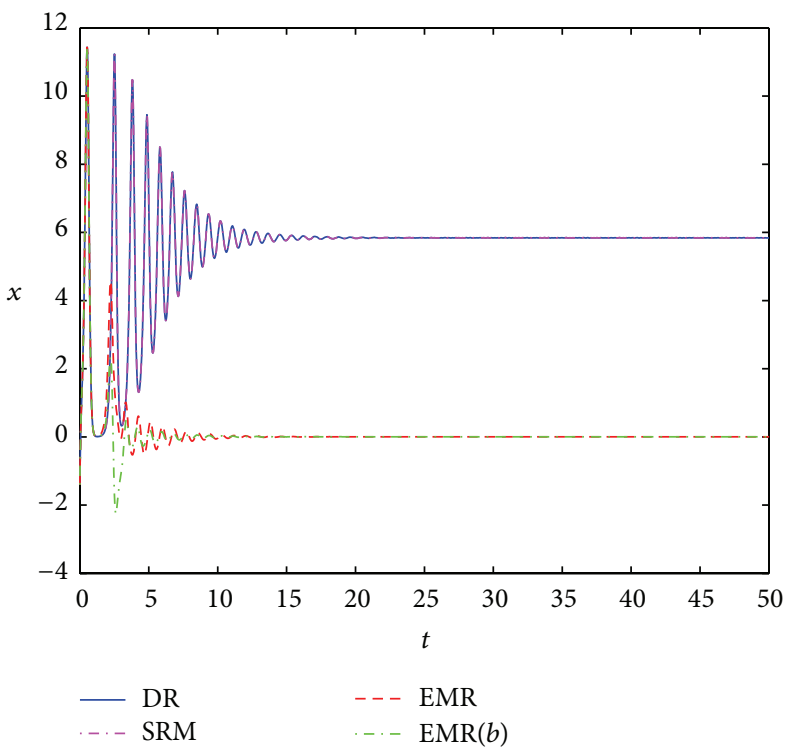

(a) $c=13.79$

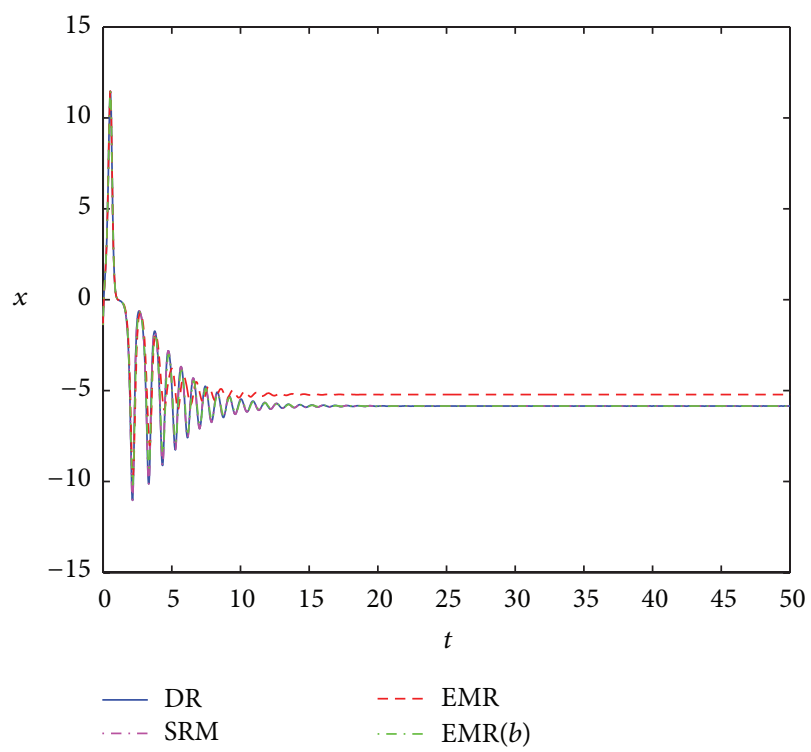

(b) $c=13.85$

FIgURE 18: The time history of the four responses.

From the bifurcation diagrams in Figure 9, it is found that the trajectories of the three responses DR, SRM, and EMR converge to the same equilibrium point in the intervals $[0,13.76) \bigcup(13.85,17.76)$. With $c=13.76$, the responses DR and SRM converge to the same equilibrium point, while the response EMR converges to another equilibrium point demonstrated in Figure 10. The responses DR and SRM converge to the same limit cycle, but the response EMR still converges to the equilibrium point for $c \in[17.76,24.04]$. Figure 11 has shown that $c=18.96$. Figure 12 shows the maximum Lyapunov exponents for the systems (9) and (11) and they enter chaos at different times. Figures 13(a) and 13(b) are their Poincaré sections. And EMR has entered chaos when DR and SRM converge to limit cycle as $c \in[24.05,24.10]$. DR, SRM, and EMR are all chaotic for $c \geq 24.11$.
Furthermore, Figure 14(a) demonstrates the maximum Lyapunov exponents for EMR and DR as $\delta_{1}=0.03$. It is shown that EMR has entered chaos with $c=24$.01. Increasing $\delta_{1}$ to 0.05 , EMR has entered chaos with $c=23.97$ in Figure 14(b). Further increasing it to 0.08 , it is obvious that EMR has entered chaos at $c=23.90$, which is displayed in Figure 15(b). But DR has not entered chaos, which is displayed in Figure 15(a). When $\delta_{1}=0.1$, EMR has been chaotic with $c=23.85$, but DR has not been chaotic, which is exhibited in Figure 16. It shows that EMR enters chaos earlier with the increase of the stochastic intensity $\delta_{1}$.

If the parameter $b$ is random in the system (9), Lorenz system with one random parameter was obtained [38]. Set $\operatorname{EMR}(b)$ for the ensemble mean response of the Lorenz system with one random parameter $b$. Then we can discuss 
TABLE 3

\begin{tabular}{lccccc}
\hline$c$ & {$[0,13.75] \bigcup(13.85,17.75]$} & $(13.75,13.85]$ & $(17.75,23.94]$ & $(23.94,24.06]$ & LC \\
DR & EP & EP & LC & LC & Chaos \\
SRM & EP & EP & LC & Chaos \\
EMR & EP & EP & EP & Chaos \\
EMR $(b)$ & EP & EP & EP & Chaos & Chaos \\
\hline
\end{tabular}

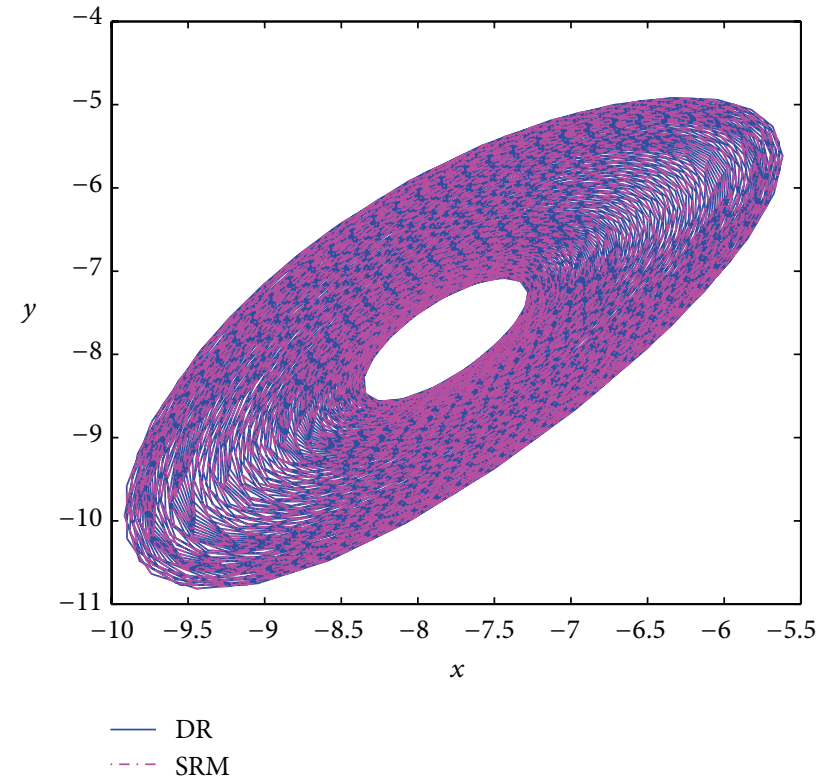

(a)

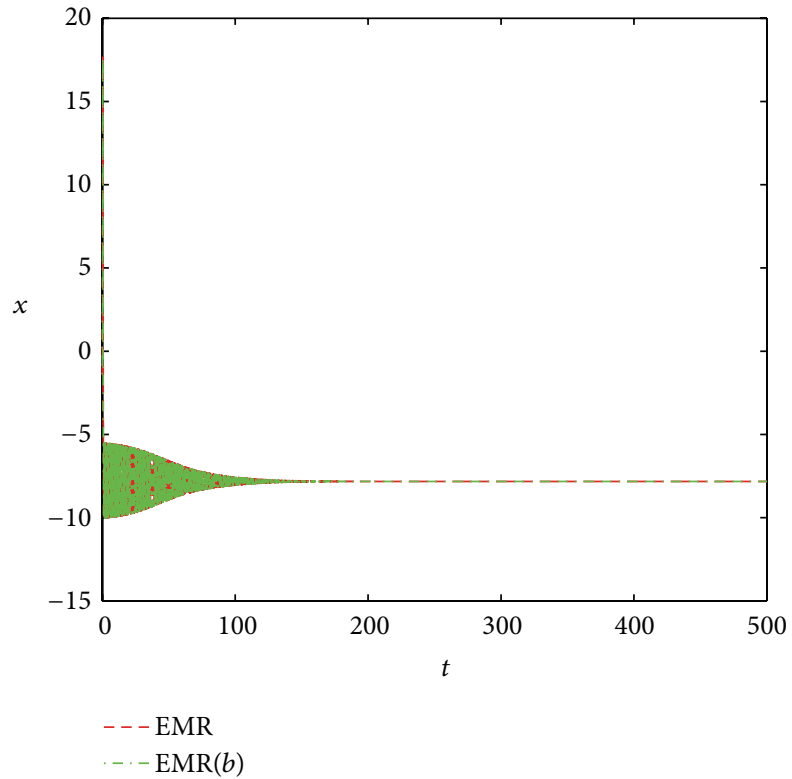

(b)

FIGURE 19: The phase portraits of the four responses with $c=23.94$.

the dynamical features of DR, SRM, EMR, and $\operatorname{EMR}(b)$. Table 3 clearly reveals this.

It is obvious that DR, SRM, $\operatorname{EMR}$, and $\operatorname{EMR}(b)$ have the same equilibrium point for $c=13.75$ in Figure 17. DR and SRM converge to one equilibrium point, while EMR and $\operatorname{EMR}(b)$ converge to another equilibrium point with $c=$ 13.79 in Figure 18(a). But DR, SRM, and $\operatorname{EMR}(b)$ have the same equilibrium point as $c=13.85$, while EMR has another equilibrium point in Figure 18(b). DR and SRM have the same periodic solution for $c=23.94$, but $\operatorname{EMR}$ and $\operatorname{EMR}(b)$ still converge to the same equilibrium point, shown in Figures 19(a) and 19(b), respectively. EMR and $\operatorname{EMR}(b)$ have been chaotic for $c=24.06$ as shown in Figures 20(b) and 20(c), while DR and SRM still converge to equilibrium point in Figure 20(a). DR, SRM, EMR, and $\operatorname{EMR}(b)$ are all chaotic for $c=24.5$.

Influenced by the random parameter $c$, the system (11) doesn't experience period state before entering chaos. The systems (11) and (9) converge to different equilibrium points in $[13.76,13.85]$. And they enter chaos at different time. Besides, the Lorenz system with multistochastic disturbances is consistent with the deterministic one very well as $\xi_{1}=\xi_{2}=$ 0 . Finally, the Lorenz system with one random parameter is a special case of the Lorenz system with multistochastic disturbances.

\section{Conclusion}

In this paper, the nonlinear chaotic system with multistochastic disturbances is studied. Firstly, the method of reducing the nonlinear chaotic system with multistochastic disturbances into an equivalent deterministic system is given according to the orthogonal polynomial approximation. Then its dynamic analysis can be achieved by discussing its equivalent deterministic system. To illustrate purposes, the Lorenz system with multistochastic disturbances is discussed. Affected by the stochastic disturbances and the stochastic intensities, the dynamic behaviors of the stochastic Lorenz system show some differences. Moreover, the Lorenz system with multistochastic disturbances is more general than such system with deterministic or one random parameter. Besides, with the increase of the stochastic intensity, the Lorenz system with multistochastic disturbances enters chaos earlier.

In the future, we will do further researches for the cases that $b$ and $c$ are correlated or both changed in the Lorenz system with multistochastic disturbances. They are interesting 


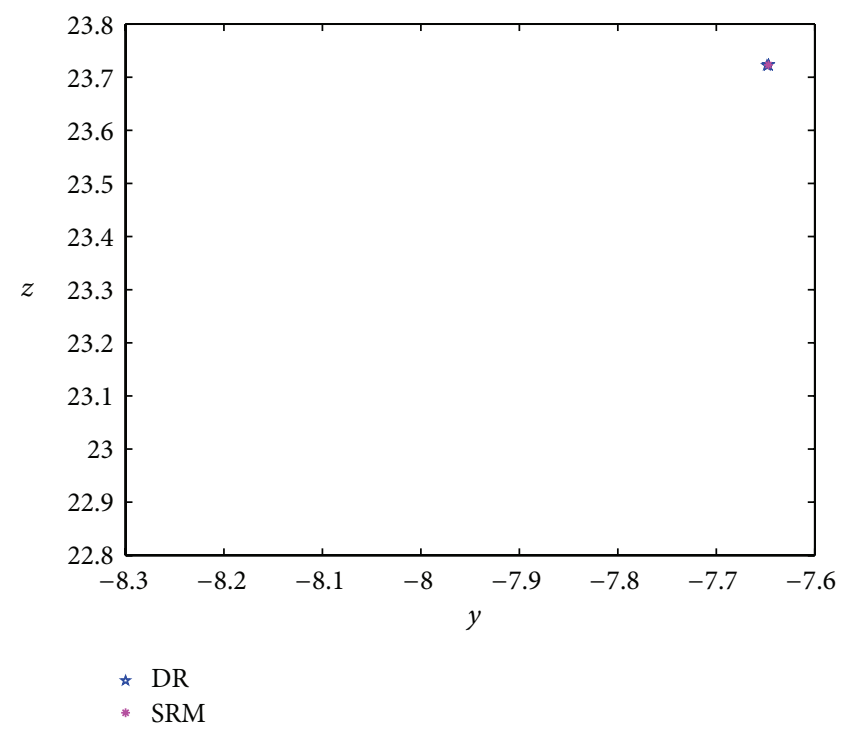

(a)

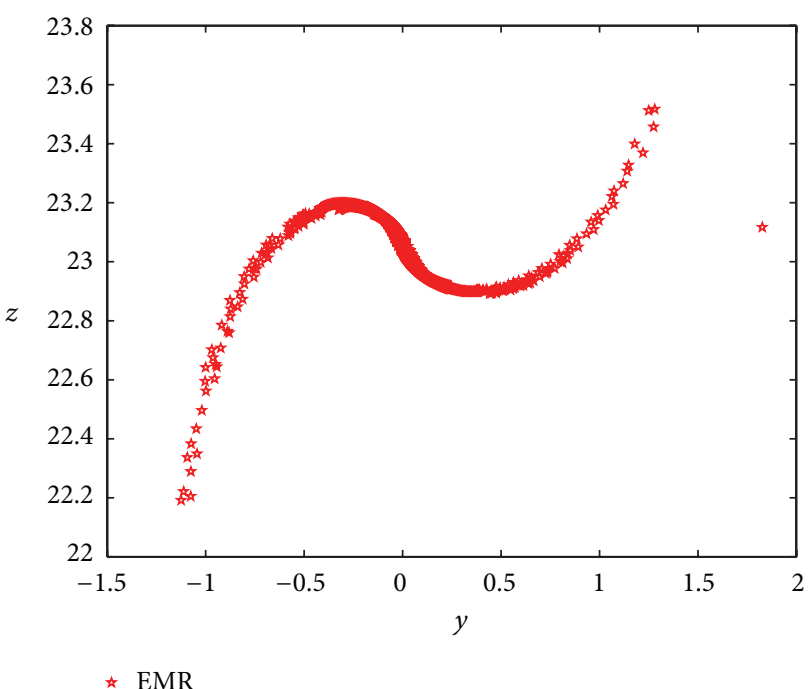

(b)

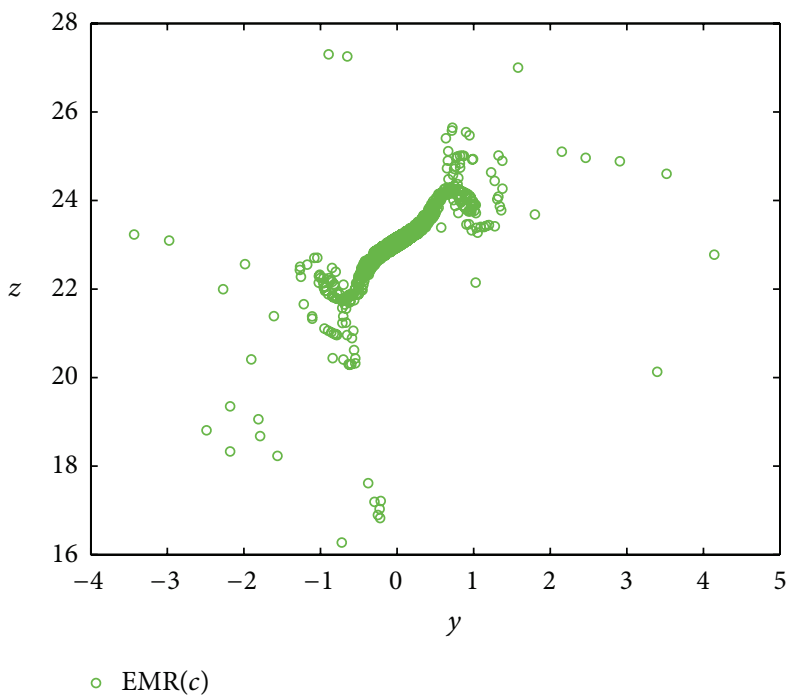

(c)

FIgURE 20: The bifurcation diagrams of the three responses.

and challenging problems and richer dynamic behaviors are expected to be found.

\section{Conflict of Interests}

The authors declare that they have no conflict of interests regarding the publication of this paper.

\section{Acknowledgments}

The authors are very grateful to the associate editor and the reviewers for their valuable comments and suggestions. This work is supported by the National Nature Science Foundation of China under Grant no. 11371049.

\section{References}

[1] J. C. Sprott, Chaos and Time-Series Analysis, Oxford University Press, New York, NY, USA, 2004.

[2] G. Chen and X. Dong, From Chaos to Order: Methodologies, Perspectives and Applications, World Scientific, Singapore, 1998.

[3] H. K. Chen, T. N. Lin, and J. H. Chen, "Dynamic analysis, controlling chaos and chaotification of a SMIB power system," Chaos, Solitons and Fractals, vol. 24, no. 5, pp. 1307-1315, 2005.

[4] Y. G. Yu and S. C. Zhang, "Hopf bifurcation analysis of the Lü system," Chaos, Solitons and Fractals, vol. 21, no. 5, pp. 12151220, 2004.

[5] E. M. Elabbasy, A. A. Elsadany, and Y. Zhang, "Bifurcation analysis and chaos in a discrete reduced Lorenz system," Applied Mathematics and Computation, vol. 228, pp. 184-194, 2014. 
[6] R. L. Stratonovich, Topics in the Theory of Random Noise, vol. 2, Gorden and Breach Science Publishers, New York, NY, USA, 1967.

[7] S. H. Crandall and W. D. Mark, Random Vibration in Mechanical Systems, Academic Press, New York, NY, USA, 1963.

[8] W. Li, W. Xu, J. Zhao, and Y. Jin, "Stochastic stability and bifurcation in a macroeconomic model," Chaos, Solitons and Fractals, vol. 31, no. 3, pp. 702-711, 2007.

[9] Y. E. Jin, W. Xu, and M. Xu, "Stochastic resonance in an asymmetric bistable system driven by correlated multiplicative and additive noise," Chaos, Solitons and Fractals, vol. 26, no. 4, pp. 1183-1187, 2005.

[10] M. Shinozuka, "Monte Carlo solution of structural dynamics," Computers \& Structures, vol. 2, no. 5-6, pp. 855-874, 1972.

[11] M. Kleiber and T. D. Hien, The Stochastic Finite Element Method: Basic Perturbation Technique and Computer Implementation, Wiley Press, 1992.

[12] R. Ghamem and P. Spans, Stochastic Finite Element: A Spectral Approach, Springer, Berlin, Germany, 1991.

[13] H. Jensen and W. D. Iwan, "Response of systems with uncertain parameters to stochastic excitation," Journal of Engineering Mechanics, vol. 118, no. 5, pp. 1012-1025, 1992.

[14] J. Li, "The expanded order system method for combined random vibration analysis," Acta Mechanica Sinica. Lixue Xuebao, vol. 28, no. 1, pp. 66-75, 1996.

[15] D. Xiu and G. E. Karniadakis, "The Wiener-Askey polynomial chaos for stochastic differential equations," SIAM Journal on Scientific Computing, vol. 24, no. 2, pp. 619-644, 2002.

[16] D. Xiu and G. E. Karniadakis, "Modeling uncertainty in flow simulations via generalized polynomial chaos," Journal of Computational Physics, vol. 187, no. 1, pp. 137-167, 2003.

[17] O. P. le Maître, H. N. Najm, R. G. Ghanem, and O. M. Knio, "Multi-resolution analysis of Wiener-type uncertainty propagation schemes," Journal of Computational Physics, vol. 197, no. 2, pp. 502-531, 2004.

[18] C. L. Pettit and P. S. Beran, "Spectral and multiresolution Wiener expansions of oscillatory stochastic processes," Journal of Sound and Vibration, vol. 294, no. 4, pp. 752-779, 2006.

[19] H. Kim, Y. Kim, and D. Yoon, "Dependence of polynomial chaos on random types of forces of KdV equations," Applied Mathematical Modelling, vol. 36, no. 7, pp. 3080-3093, 2012.

[20] T. Fang, X. L. Leng, and C. Q. Song, "Chebyshev polynomial approximation for dynamical response problem of random system," Journal of Sound and Vibration, vol. 266, no. 1, pp. 198206, 2003.

[21] T. Fang, X. Leng, X. Ma, and G. Meng, “ $\lambda$-PDF and Gegenbauer polynomial approximation for dynamic response problems of random structures," Acta Mechanica Sinica, vol. 20, no. 3, pp. 292-298, 2004.

[22] S. J. Ma, W. Xu, and T. Fang, "Analysis of period-doubling bifurcation in double-well stochastic Duffing system via Laguerre polynomial approximation," Nonlinear Dynamics, vol. 52, no. 3 , pp. 289-299, 2008.

[23] S. J. Ma and W. Xu, "Period-doubling bifurcation in an extended van der Pol system with bounded random parameter," Communications in Nonlinear Science and Numerical Simulation, vol. 13, no. 10, pp. 2256-2265, 2008.

[24] W. Li, W. Xu, J. Zhao, and S. Ma, "Stochastic optimal control of first-passage failure for coupled Duffing-van der Pol system under Gaussian white noise excitations," Chaos, Solitons and Fractals, vol. 25, no. 5, pp. 1221-1228, 2005.
[25] S. J. Ma, The orthogonal polynomial approximation analysis of the stochastic bifurcation [M.S. thesis], Northwestern Polytechnical University, 2005, (Chinese).

[26] X. Z. Wang and D. R. Guo, Special Functions, World Scientific Publishing, Singapore, 1989.

[27] X. Li, K. E. Chlouverakis, and D. Xu, "Nonlinear dynamics and circuit realization of a new chaotic flow: a variant of Lorenz, Chen and Lü," Nonlinear Analysis: Real World Applications, vol. 10, no. 4, pp. 2357-2368, 2009.

[28] S. Dadras, H. R. Momeni, and G. Qi, "Analysis of a new 3D smooth autonomous system with different wing chaotic attractors and transient chaos," Nonlinear Dynamics, vol. 62, no. 1-2, pp. 391-405, 2010.

[29] S. Jafari, J. C. Sprott, and S. M. R. H. Golpayegani, "Elementary quadratic chaotic flows with no equilibria," Physics Letters A, vol. 377, no. 9, pp. 699-702, 2013.

[30] A. Algaba, F. Fernández-Sánchez, M. Merino, and A. J. Rodríguez-Luis, "Chens attractor exists if Lorenz repulsor exists: the Chen system is a special case of the Lorenz system," Chaos, vol. 23, Article ID 033108, 2013.

[31] A. Algaba, F. Fernández-Sánchez, M. Merino, and A. J. Rodríguez-Luis, "The Lü system is a particular case of the Lorenz system," Physics Letters A, vol. 377, no. 39, pp. 2771-2776, 2013.

[32] E. N. Lorenz, "Deterministic nonperiodic flow," Journal of the Atmospheric Sciences, vol. 20, no. 2, pp. 130-141, 1963.

[33] M. Moghtadaei and M. R. Hashemi Golpayegani, "Complex dynamic behaviors of the complex Lorenz system," Scientia Iranica, vol. 19, no. 3, pp. 733-738, 2012.

[34] L. S. Tee and Z. Salleh, "Dynamical analysis of a modified Lorenz system," Journal of Mathematics, vol. 2013, Article ID 820946, 8 pages, 2013.

[35] E. Knobloch, "On the statistical dynamics of the Lorenz model," Journal of Statistical Physics, vol. 20, no. 6, pp. 695-709, 1979.

[36] T. Song and C. Cattani, "Fast detection of weak singularities in a chaotic signal using Lorenz system and the bisection algorithm," Mathematical Problems in Engineering, vol. 2012, Article ID 102848, 10 pages, 2012.

[37] J. Weiland, J. P. Mondt, and R. A. Gerwin, "Complex Lorenz system for collisional inhomogeneous plasmas," Physical Review A, vol. 34, no. 1, pp. 647-650, 1986.

[38] X. S. Lin, Y. G. Yu, and H. Wang, "Dynamics analysis of the stochastic Lorenz system," in Proceedings of the 4th International Workshop on Chaos-Fractals Theories and Applications (IWCFTA '11), pp. 32-36, October 2011. 


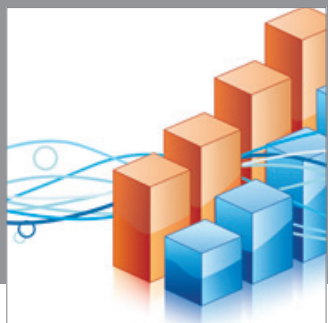

Advances in

Operations Research

mansans

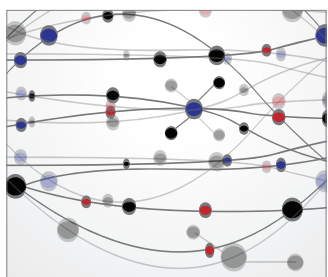

The Scientific World Journal
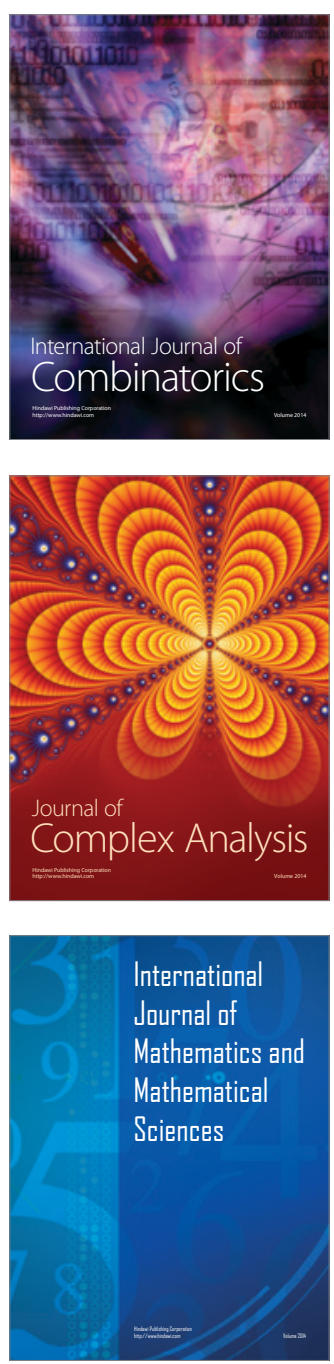
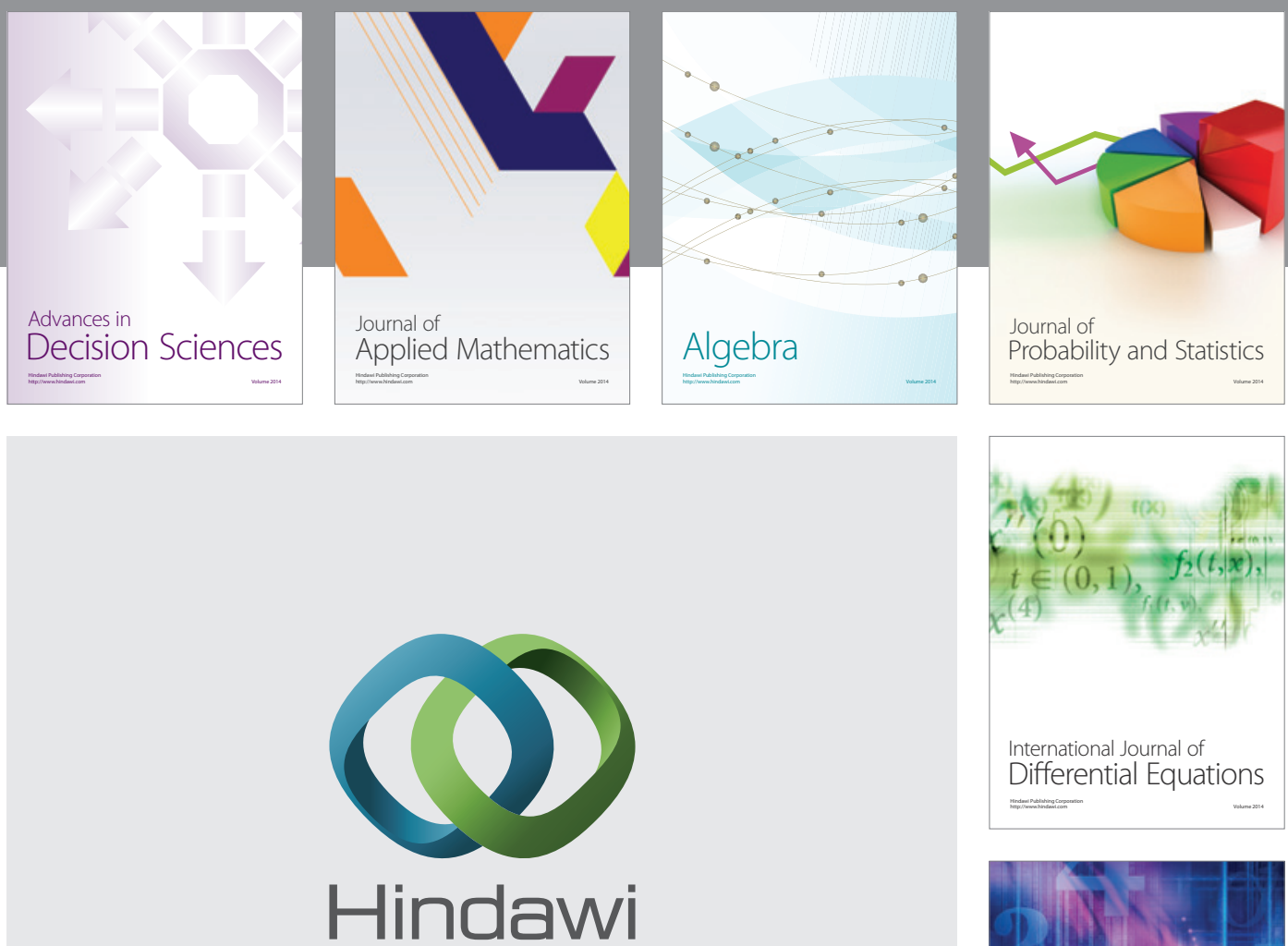

Submit your manuscripts at http://www.hindawi.com
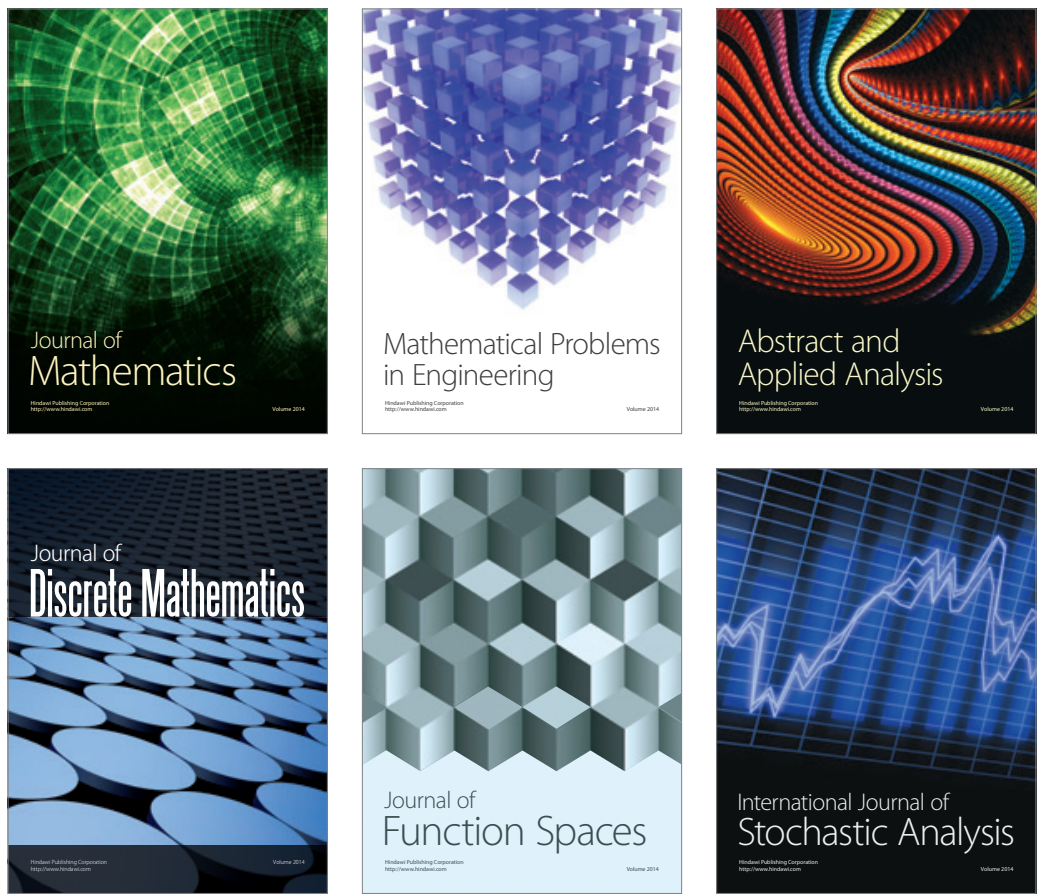

Journal of

Function Spaces

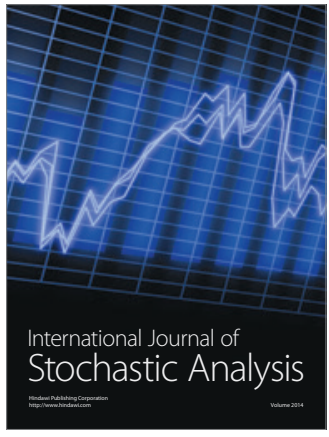

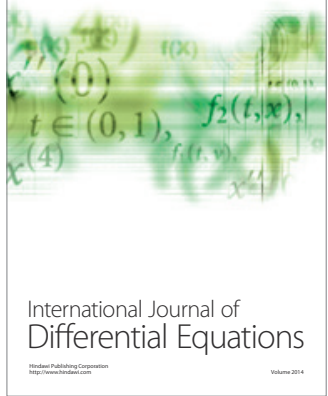
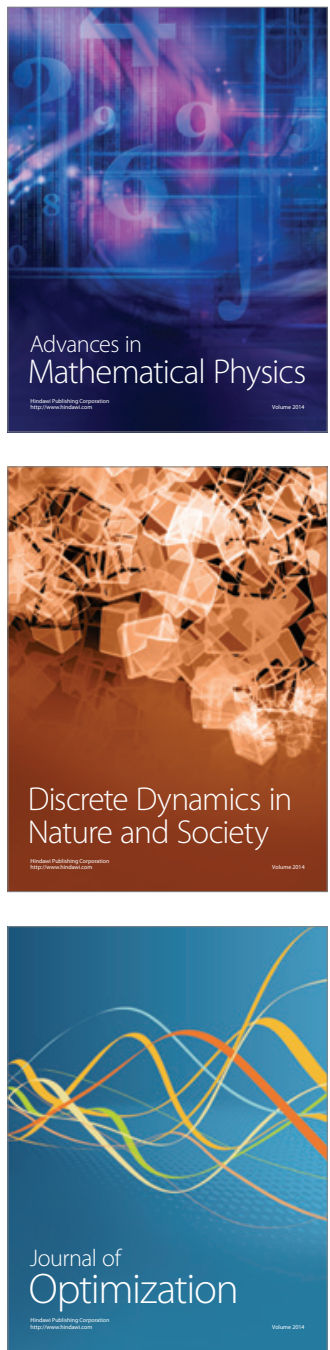